\title{
Breast cancer risk factors in relation to molecular subtypes in breast cancer patients from Kenya
}

Shahin Sayed ${ }^{1,2^{*}}$ (D), Shaogi Fan ${ }^{3}$, Zahir Moloo ${ }^{1}$, Ronald Wasike ${ }^{1}$, Peter Bird ${ }^{4}$, Mansoor Saleh ${ }^{1}$, Asim Jamal Shaikh', Jonine D. Figueroa ${ }^{5}$, Richard Naidoo ${ }^{2}$, Francis W. Makokha ${ }^{6}$, Kevin Gardner ${ }^{7}$, Raymond Oigara ${ }^{8,9}$, Faith Wambui Njoroge ${ }^{10}$, Pumza Magangane ${ }^{11}$, Miriam Mutebi ${ }^{1}$, Rajendra Chauhan ${ }^{1}$, Sitna Mwanzi ${ }^{1}$, Dhirendra Govender ${ }^{2,12 \dagger}$ and Xiaohong R. Yang ${ }^{3 \dagger}$

\begin{abstract}
Background: Few studies have investigated risk factor heterogeneity by molecular subtypes in indigenous African populations where prevalence of traditional breast cancer (BC) risk factors, genetic background, and environmental exposures show marked differences compared to European ancestry populations.

Methods: We conducted a case-only analysis of 838 pathologically confirmed BC cases recruited from 5 groups of public, faith-based, and private institutions across Kenya between March 2012 to May 2015. Centralized pathology review and immunohistochemistry (IHC) for key markers (ER, PR, HER2, EGFR, CK5-6, and Ki67) was performed to define subtypes. Risk factor data was collected at time of diagnosis through a questionnaire. Multivariable polytomous logistic regression models were used to determine associations between BC risk factors and tumor molecular subtypes, adjusted for clinical characteristics and risk factors.

Results: The median age at menarche and first pregnancy were 14 and 21 years, median number of children was 3 , and breastfeeding duration was 62 months per child. Distribution of molecular subtypes for luminal A, luminal B, HER2-enriched, and triple negative (TN) breast cancers was 34.8\%, 35.8\%, 10.7\%, and 18.6\%, respectively. After adjusting for covariates, compared to patients with ER-positive tumors, ER-negative patients were more likely to have higher parity $(\mathrm{OR}=2.03,95 \% \mathrm{Cl}=(1.11,3.72), p=0.021$, comparing $\geq 5$ to $\leq 2$ children). Compared to patients with luminal A tumors, luminal B patients were more likely to have lower parity $(\mathrm{OR}=0.45,95 \% \mathrm{Cl}=0.23$, $0.87, p=0.018$, comparing $\geq 5$ to $\leq 2$ children); HER2-enriched patients were less likely to be obese $(\mathrm{OR}=0.36$, $95 \% \mathrm{Cl}=0.16,0.81, p=0.013$ ) or older age at menopause $(\mathrm{OR}=0.38,95 \% \mathrm{Cl}=0.15,0.997, p=0.049)$. Body mass index (BMI), either overall or by menopausal status, did not vary significantly by ER status. Overall, cumulative or average breastfeeding duration did not vary significantly across subtypes.
\end{abstract}

\footnotetext{
* Correspondence: shaheen.sayed@aku.edu

${ }^{\dagger}$ Dhirendra Govender and Xiaohong R. Yang are joint senior authors.

'Department of Pathology, Aga Khan University, Nairobi, Kenya

${ }^{2}$ University of Cape Town, Cape Town, South Africa

Full list of author information is available at the end of the article
}

\section{$\triangle B M C$}

(c) The Author(s). 2021 Open Access This article is licensed under a Creative Commons Attribution 4.0 International License, which permits use, sharing, adaptation, distribution and reproduction in any medium or format, as long as you give appropriate credit to the original author(s) and the source, provide a link to the Creative Commons licence, and indicate if changes were made. The images or other third party material in this article are included in the article's Creative Commons licence, unless indicated otherwise in a credit line to the material. If material is not included in the article's Creative Commons licence and your intended use is not permitted by statutory regulation or exceeds the permitted use, you will need to obtain permission directly from the copyright holder. To view a copy of this licence, visit http://creativecommons.org/licenses/by/4.0/ The Creative Commons Public Domain Dedication waiver (http://creativecommons.org/publicdomain/zero/1.0/) applies to the data made available in this article, unless otherwise stated in a credit line to the data. 
Conclusions: In Kenya, we found associations between parity-related risk factors and ER status consistent with observations in European ancestry populations, but differing associations with BMI and breastfeeding. Inclusion of diverse populations in cancer etiology studies is needed to develop population and subtype-specific risk prediction/ prevention strategies.

Keywords: Breast cancer, Molecular subtypes, Risk factors, Kenya, Sub-Saharan Africa

\section{Background}

Women in Africa have lower incidence rates of breast cancer (BC) than women in developed countries (agestandardized rates (ASR) per 100,000 of 36 vs. 74), but higher mortality rates (ASR of 17 vs. 15) [1]. Furthermore, there is variation in the relative survival (RS) from $\mathrm{BC}$ by stage and country-level human development index (HDI) in sub-Saharan Africa (SSA) with the 5-year RS after breast cancer diagnosis in Mauritius at $83.2 \%$ and the lowest in Uganda at $12.1 \%$, while it ranges between 40.1 and $64 \%$ in Kenya as per data abstracted from the Eldoret and Nairobi Cancer Registries, respectively [2]. Furthermore, survival differences in SSA remain for any given breast cancer stage with the lowest 3 -year breast cancer-specific survival observed in Nigeria at $38 \%$ compared with $68 \%$ in Black women from Namibia, thus underlying as yet unexplained risks with survival [3]. In Kenya, country figures indicate that BC is the most frequently diagnosed cancer among women, representing $20.8 \%$ of all cancer cases, and the second most common cause from cancer mortality [4].

Although advanced stage at presentation, lack of awareness about $\mathrm{BC}$ and limited access to available screening and treatment options [5] are contributing factors to disparate mortality rates, whether incidence for more aggressive breast cancers are higher in African women remains controversial. Women of African descent present with $\mathrm{BCs}$ a decade earlier than their Caucasian counterparts [6,3], and despite correcting for risk factor distribution, their tumors still tend to be estrogen receptor (ER) negative [7], suggesting the interplay of other biologic and genetic differences that remain largely unexplored.

Breast cancer can be divided into several molecular subtypes based on gene expression profiling analysis, which are subsequently corroborated by a panel of immunohistochemical (IHC) markers including ER, progesterone receptor (PR), human epidermal growth receptor factor 2 (HER2), proliferation marker Ki-67, cytokeratin (CK) 5/6, and epidermal growth factor receptor (EGFR). Epidemiologic studies have demonstrated that $\mathrm{BC}$ risk associated with established risk factors, including genetic and environment/lifestyle factors, differ for different breast cancer subtypes [8], which highlights the importance of developing subtype-specific risk prediction and prevention strategies [9]. Overwhelmingly, these breast cancer prediction models have been derived from European ancestry women and some studies have noted poor performance in African women [10]. This is likely explained by the differential associations of risk factors such as parity and obesity for ER-positive and ERnegative cancers and higher frequencies of ER-negative cancers among African women. In addition, the prevalence of breast cancer risk factors, including genetic background and environmental exposures, show marked differences between indigenous African and European and even African American women. Notably, women in African countries are more likely to have high exposures to infectious agents (malaria and other parasites), and a low prevalence of traditional BC risk factors (including low or late parity, lack of breastfeeding, obesity, and exogenous hormone use), which may contribute to differences in the risk of different BC subtypes. Furthermore, there are great variations in genetic structure and exposures as well as breast cancer subtype distributions across different African populations [11, 7, 12]. Therefore, studies in diverse indigenous African populations will allow for a broader capture of associations between risk factors and tumor subtypes, particularly for exposures and subtypes that are in general very rare but are prevalent in African populations. Findings from these studies will improve our understanding of risk factor heterogeneity and our ability to develop risk prediction models that are better tailored for specific African populations.

Here, in this study, using carefully annotated risk factor and pathology data collected from $838 \mathrm{BC}$ patients enrolled from multiple hospitals across Kenya, we aimed to evaluate distributions of established $\mathrm{BC}$ risk factors across BC subtypes.

\section{Methods}

\section{Study population and risk factor data}

The study has been previously described but in brief, 838 pathologically confirmed $\mathrm{BC}$ cases were collected across Kenya between March 2012 and May 2015 [13]. There were 15 hospital/health facilities which we grouped into 5 network/regional facilities: Aga Khan University (AKU) hospitals (including AKU hospitals at Kisumu, Mombasa, and Nairobi), AIC Kijabe Hospital, Nyeri Provincial General Hospital (PGH), St Mary's Mission Hospital (Nairobi), and others (Supplementary 
Table 1). The grouping was based on whether public, faith-based or private institutions. Institutional ethics approval was obtained. Socio-demographic, clinical, reproductive, and known breast cancer risk factor data were collected using a standardized questionnaire.

\section{Pathology, immunohistochemical data, and molecular subtypes}

Pathologic characteristics including histologic grade, histologic tumor type, tumor size, lymph node stage, lymphovascular invasion, and ER/PR/HER2 status were extracted from the clinical database. Central pathology review and IHC for ER/PR/HER2 of all breast carcinoma tissue were done at AKU Hospital, Nairobi, and interpreted by SS and ZM. AKU Pathology department is a College of American Pathologists accredited laboratory and as such enrolls in proficiency testing schemes for breast biomarkers. Additional slides were cut at $5 \mu \mathrm{m}$ and subjected to IHC stains for EGFR, CK5/6, and Ki67 (Dako Monoclonal mouse anti-human antibodies were used; wild type EGFR polyclonal antibody in a dilution of 1:200, CK5/6 clone D5/16 B4 ready to use, Ki-67 Clone MIB-1, ready to use) according to the manufacturer specifications as previously described [13], with appropriate control tissues included, and stained on the DAKO Autostainer link instrument.

ER and PR tumor expression were considered positive by $\mathrm{IHC}$ with $\geq 1 \%$ nuclear staining. HER2 expression was determined by IHC and fluorescence in situ hybridization (FISH), the latter in case of an equivocal HER2 IHC result. An IHC score of $3+$ or a FISHpositive test result was defined as HER2-positive [14]. Ki-67 was considered high if $20 \%$ or more of the cells showed nuclear staining based on St Gallen recommendation [15].

We used Ki-67 status (low/high) to discriminate luminal A and B and used tumor grade as a surrogate for patients with missing Ki-67 [16]. For EGFR and CK5/6, a result was considered positive for any amount of cytoplasmic or membranous staining in any percentage of tumor cells as per the recommendations from the British Columbia study for defining the Basal subtype of breast cancer [17].

Molecular subtypes were defined based on previous clinically validated guidelines [18] (Fig. 1): luminal A: ER+ and/or PR+, HER2-, and low Ki-67/histologic grade (I or II); luminal B-HER2+: ER+ and/or PR+, and HER2+; luminal B-high proliferative: ER+ or PR+, HER2 -, and high Ki-67/histologic grade (III); HER2-enriched: ER-, PR-, and HER2+; and triple-negative (TN): ER-, PR-, HER2 (Fig. 1). Due to the small sample size, in primary subtype analysis, we grouped the two luminal B subtypes into a single subtype for risk factor associations. For patients with EGFR and CK5/6 data available, we further stratified TN patients into core-basal like (CK5/6+ and/or EGFR+) and five negative (CK5/6- and EGFR-).

\section{Statistical analysis}

Distributions of breast cancer risk factors, including sociodemographic, reproductive, and tumor pathologic characteristics in the overall study population and by hospital groups, were assessed using the chi-squared test or Fisher's exact test. Multivariable polytomous logistic regression models were used to determine associations

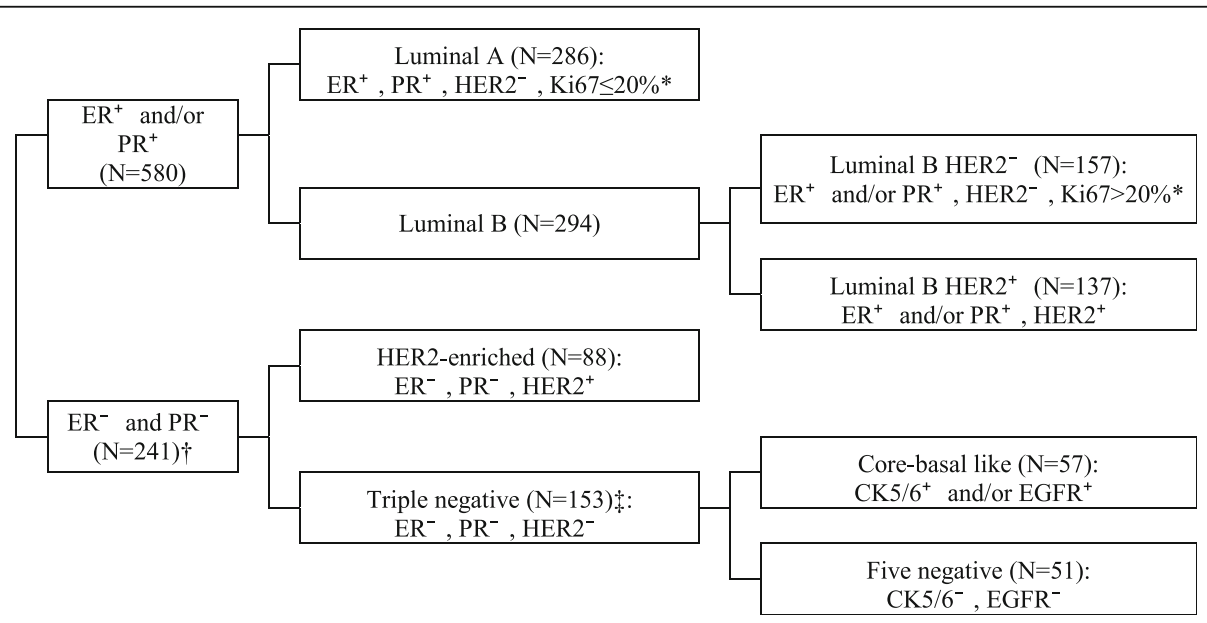

Fig. 1 Breast tumor subtype definition in Kenyan breast cancer patients $(\mathrm{N}=838) .{ }^{*}$ Tumor grade was used to determine tumor subtypes in the absence of ki67: if tumor grade is low or intermediate, define tumor subtype as "Luminal A"; if tumor grade is high, define tumor subtype as "Luminal B HER2"." †Seventeen cases are not included due to their missing HER2 status. \#Forty-five cases are not included due to their missing CK5/6 and EGFR status. CK5/6, cytokeratin 5/6; EGFR, epidermal growth factor receptor; ER, estrogen receptor; HER2, human epidermal growth factor receptor-2; PR, progesterone receptor 
between $\mathrm{BC}$ risk factors and tumor molecular subtypes (ER status or luminal A-like as the reference).

All regression models were fully adjusted for the same covariates (except for where noted): age at diagnosis, BMI, age at menarche, age at first pregnancy, number of children, averaged breastfeeding duration, age at menopause, family history of breast cancer in 1st degree female relatives, highest education level, and occupation. A two-tailed $P$ value less than 0.05 was considered statistically significant. All analyses were performed with SAS v9.4 statistical software (SAS Institute Inc.).

\section{Results}

\section{Descriptive analysis of sociodemographic and} reproductive characteristics

There were 838 invasive breast cancer cases with complete data on ER and PR status after exclusion of DCIS cases $(n=21)$ and cases without any data for tumor subtype $(\mathrm{n}=8)$. Fifty-four percent of patients were diagnosed under 50 years of age, $69 \%$ had BMI $\geq 25 \mathrm{~kg} / \mathrm{m}^{2}$ at diagnosis and $61 \%$ lived in rural areas. Our study population was also characteristic for late age at menarche $(\geq$ 13 years, 92\%), young age at first pregnancy $(<25$ years, $70 \%)$, having 3 or more children (68\%), high prevalence in breastfeeding (95\%), and long breastfeeding duration ( $\geq 1$ year per child, $80 \%$ ) (Table 1 ).

Compared to patients admitted to the other 4 hospital groups, AKU patients were more likely to be overweight or obese $(79 \%)$, have tertiary education level $(45 \%)$, start the first pregnancy $\geq 25$ years $(35 \%)$, have $<3$ children (39\%), and have shorter breastfeeding duration per child, which is as expected given that AKU is a private health facility, and compared to the others, patients are generally from a higher socioeconomic status.

\section{Distributions of tumor subtypes and pathologic characteristics in the overall study population and by hospitals}

The distribution of tumor subtypes defined by IHC markers is presented in Fig. 1 and Table 2. Overall, $69.5 \%, 59.4 \%$, and $27.4 \%$ of patients were $\mathrm{ER}+, \mathrm{PR}+$, and HER2+, respectively. After classifying BC into molecular subtypes, $34.8 \%$, 35.8\%, $10.7 \%$, and $18.6 \%$ of patients had luminal A, luminal B, HER2-enriched, and TN breast cancers, respectively. More than $90 \%$ of patients had tumors larger than $2 \mathrm{~cm}(2-<5 \mathrm{~cm}, 53.5 \%$; $\geq 5 \mathrm{~cm}, 38.9 \%)$ and had intermediate-to-high tumor grade (intermediate, 45.9\%; high, 49.1\%). Sixty-one percent of tumors showed lymphovascular invasion. Nearly half of patients received definitive surgery, either lumpectomy or mastectomy, among which $91 \%$ had stage II or higher disease and for those cases with lymph node metastases, 39.5\% were positive for extra-nodal extension. AKU patients were more likely to have small $(\leq 2 \mathrm{~cm})$ and early-stage tumors $(P<0.01)$. Patients admitted to Kijabe and Nyeri hospitals had higher proportions of tumors with lymphovascular invasion: $71.4 \%$ and $69.1 \%$, respectively. There was no statistical difference in distributions of patient molecular subtypes (defined by ER, PR, and HER2) across hospitals $(P=0.08)$.

\section{Associations between breast cancer risk factors and tumor subtypes ER, PR, and HER2}

Results of adjusted associations between risk factors and ER status are shown in Table 3. Compared to ER-positive patients, ER-negative patients were more likely to have higher parity $(\mathrm{OR}=2.03,95 \%$ $\mathrm{CI}=1.11,3.72, \mathrm{P}_{\text {trend }}=0.021$, comparing $\geq 5$ to $\leq 2$ children). ER-negative patients were also more likely to have longer cumulative breastfeeding duration $(\mathrm{OR}=2.38,95 \% \mathrm{CI}=1.33$, 4.24; comparing $\geq 62$ to $<39$ months); however, these positive associations became insignificant after adjusting for a number of children. In fact, analyzing parity and breastfeeding variables together showed that the association was driven by parity (Table 3 ). In addition, the average duration of breastfeeding per child did not vary significantly by ER. Overall, we observed similar associations for PR to those for ER (Supplementary Table 2). BMI, either overall or by menopausal status, did not significantly vary by ER or PR status. When stratified by HER2 status, we found that, compared to HER2-negative patients, HER2-positive patients were less likely to be obese $(\mathrm{OR}=0.58$, 95\% CI $\left.=0.34,0.97, \mathrm{P}_{\text {trend }}=0.038\right)$, especially among postmenopausal women $(\mathrm{OR}=0.26,95 \% \mathrm{CI}$ $\left.=0.10,0.62, \mathrm{P}_{\text {trend }}=0.0026\right)$ (Supplementary Table 2). Similar results were observed when we restricted to early-stage patients $(\mathrm{OR}=0.76,95 \% \mathrm{CI}=0.59$, $0.98, \mathrm{P}_{\text {trend }}=0.038$ ) suggesting that the association was unlikely to be due to the reverse causation.

Given that several risk factors and clinical variables varied by hospital groups (Tables 1 and 2), we next tested whether the observed associations varied among patients admitted to different hospital groups. In this analysis, we selected five key risk factors (i.e., BMI, age at first pregnancy, number of children, and mean breastfeeding duration per child, combined number of children and cumulative breastfeeding duration) and stratified their associations with ER or HER2 (for BMI) status by five hospital groups (Fig. 2 and Supplementary Figure 1; Supplementary Table 3 and 4). With the exception of Nyeri, the associations with ER were fairly consistent across other hospitals for age at first birth, parity, and breastfeeding (Fig. 2). In contrast, the association between BMI and HER2 appeared to be driven by AKU 
Table 1 Distributions of breast cancer risk factors in Kenyan breast cancer patients, overall and by hospitals ( $N=838)$

\begin{tabular}{|c|c|c|c|c|c|c|c|c|c|c|c|c|c|}
\hline & \multirow{2}{*}{\multicolumn{2}{|c|}{ Overall ( $n=838)$}} & \multicolumn{11}{|l|}{ Hospitals } \\
\hline & & & \multicolumn{2}{|c|}{ AKU $(n=350,42 \%)$} & \multicolumn{2}{|c|}{ Kijabe $(n=105,13 \%)$} & \multicolumn{2}{|c|}{$\begin{array}{l}\text { Nyeri } \\
(n=110, \\
13 \%)\end{array}$} & \multicolumn{2}{|c|}{$\begin{array}{l}\text { St } \\
\text { Mary's } \\
(n=122, \\
15 \%)\end{array}$} & \multicolumn{2}{|c|}{$\begin{array}{l}\text { Others } \\
(n=151, \\
18 \%)\end{array}$} & \multirow[b]{2}{*}{$\mathrm{P}^{*}$} \\
\hline & $\mathrm{N}$ & $\%$ & $\mathrm{~N}$ & $\%$ & $\mathrm{~N}$ & $\%$ & $\mathrm{~N}$ & $\%$ & $\mathrm{~N}$ & $\%$ & $\mathrm{~N}$ & $\%$ & \\
\hline \multicolumn{14}{|l|}{ Demographic } \\
\hline \multicolumn{14}{|l|}{ Age at diagnosis/year } \\
\hline $20-29$ & 32 & 3.8 & 17 & 4.9 & 2 & 1.9 & 2 & 1.9 & 5 & 4.1 & 6 & 4.0 & 0.11 \\
\hline 30-39 & 177 & 21.2 & 64 & 18.3 & 23 & 21.9 & 22 & 20.4 & 34 & 28.1 & 34 & 22.5 & \\
\hline $40-49$ & 242 & 29.0 & 100 & 28.7 & 37 & 35.2 & 34 & 31.5 & 35 & 28.9 & 36 & 23.8 & \\
\hline $50-59$ & 211 & 25.3 & 105 & 30.1 & 26 & 24.8 & 23 & 21.3 & 19 & 15.7 & 38 & 25.2 & \\
\hline$\geq 60$ & 172 & 20.6 & 63 & 18.1 & 17 & 16.2 & 27 & 25.0 & 28 & 23.1 & 37 & 24.5 & \\
\hline Mean (SD) & $49.2(12.8)$ & $49.0(11.2)$ & $48.4(11.3)$ & $50.5(13.5)$ & $48.7(15.1)$ & 49.7 (12.9) & & & & & & & \\
\hline Median (IQR) & $48(39,57)$ & $49(40,56)$ & $47(41,57)$ & $49(41,60)$ & $45(37,58)$ & $49(39,59)$ & & & & & & & \\
\hline Missing & 4 & & 1 & & 0 & & 2 & & 1 & & 0 & & \\
\hline \multicolumn{14}{|l|}{$\mathrm{BMI} / \mathrm{kg} / \mathrm{m}^{2}$} \\
\hline Normal $(<25.0)$ & 210 & 31.3 & 67 & 21.3 & 35 & 40.2 & 25 & 39.7 & 36 & 40.4 & 47 & 40.2 & $<0.0001$ \\
\hline Overweight (25.0-29.9) & 264 & 39.3 & 126 & 40.0 & 33 & 37.9 & 28 & 44.4 & 37 & 41.6 & 40 & 34.2 & \\
\hline Obese ( $\geq 30.0)$ & 197 & 29.4 & 122 & 38.7 & 19 & 21.8 & 10 & 15.9 & 16 & 18.0 & 30 & 25.6 & \\
\hline Missing & 167 & & 35 & & 18 & & 47 & & 33 & & 34 & & \\
\hline \multicolumn{14}{|c|}{ Family history of breast cancer in first-degree female relatives } \\
\hline No & 773 & 92.2 & 314 & 89.7 & 100 & 95.2 & 99 & 90.0 & 119 & 97.5 & 141 & 93.4 & 0.036 \\
\hline Yes & 65 & 7.8 & 36 & 10.3 & 5 & 4.8 & 11 & 10.0 & 3 & 2.5 & 10 & 6.6 & \\
\hline \multicolumn{14}{|l|}{ Occupation } \\
\hline Farmer & 253 & 30.3 & 47 & 13.4 & 43 & 41.0 & 82 & 74.5 & 39 & 32.5 & 42 & 27.8 & $<0.0001$ \\
\hline Employed worker & 195 & 23.3 & 138 & 39.4 & 16 & 15.2 & 5 & 4.5 & 10 & 8.3 & 26 & 17.2 & \\
\hline Trader & 155 & 18.5 & 66 & 18.9 & 24 & 22.9 & 2 & 1.8 & 35 & 29.2 & 28 & 18.5 & \\
\hline Housewife & 156 & 18.7 & 68 & 19.4 & 19 & 18.1 & 8 & 7.3 & 27 & 22.5 & 34 & 22.5 & \\
\hline Casual worker & 32 & 3.8 & 11 & 3.1 & 3 & 2.9 & 4 & 3.6 & 8 & 6.7 & 6 & 4.0 & \\
\hline Other & 45 & 5.4 & 20 & 5.7 & 0 & 0.0 & 9 & 8.2 & 1 & 0.8 & 15 & 9.9 & \\
\hline Missing & 2 & & 0 & & 0 & & 0 & & 2 & & 0 & & \\
\hline \multicolumn{14}{|l|}{ Highest education level } \\
\hline None & 257 & 30.7 & 54 & 15.4 & 41 & 39.0 & 44 & 40.0 & 57 & 46.7 & 61 & 40.4 & $<0.0001$ \\
\hline Primary & 163 & 19.5 & 40 & 11.4 & 22 & 21.0 & 29 & 26.4 & 36 & 29.5 & 36 & 23.8 & \\
\hline Secondary & 209 & 24.9 & 99 & 28.3 & 24 & 22.9 & 31 & 28.2 & 21 & 17.2 & 34 & 22.5 & \\
\hline Tertiary & 209 & 24.9 & 157 & 44.9 & 18 & 17.1 & 6 & 5.5 & 8 & 6.6 & 20 & 13.2 & \\
\hline \multicolumn{14}{|l|}{ Place of residence } \\
\hline Rural & 511 & 61.0 & 173 & 49.4 & 79 & 75.2 & 99 & 90.0 & 65 & 53.3 & 95 & 62.9 & $<0.0001$ \\
\hline Urban & 327 & 39.0 & 177 & 50.6 & 26 & 24.8 & 11 & 10.0 & 57 & 46.7 & 56 & 37.1 & \\
\hline \multicolumn{14}{|l|}{ Exposure to smokingt } \\
\hline Never exposed & 477 & 56.9 & 248 & 70.9 & 63 & 60.0 & 39 & 35.5 & 63 & 51.6 & 64 & 42.4 & $<0.0001$ \\
\hline Exposed & 361 & 43.1 & 102 & 29.1 & 42 & 40.0 & 71 & 64.5 & 59 & 48.4 & 87 & 57.6 & \\
\hline \multicolumn{14}{|l|}{ Alcohol use } \\
\hline No & 760 & 90.7 & 304 & 86.9 & 98 & 93.3 & 109 & 99.1 & 115 & 94.3 & 134 & 88.7 & 0.0009 \\
\hline Yes & 78 & 9.3 & 46 & 13.1 & 7 & 6.7 & 1 & 0.9 & 7 & 5.7 & 17 & 11.3 & \\
\hline \multicolumn{14}{|l|}{ Ethnicity } \\
\hline Bantu & 656 & 78.3 & 266 & 76.0 & 92 & 87.6 & 109 & 99.1 & 106 & 86.9 & 83 & 55.0 & $<0.0001$ \\
\hline Nilote & 141 & 16.8 & 65 & 18.6 & 5 & 4.8 & 0 & 0.0 & 10 & 8.2 & 61 & 40.4 & \\
\hline Cushite/Mixed & 41 & 4.9 & 19 & 5.4 & 8 & 7.6 & 1 & 0.9 & 6 & 4.9 & 7 & 4.6 & \\
\hline
\end{tabular}


Table 1 Distributions of breast cancer risk factors in Kenyan breast cancer patients, overall and by hospitals ( $N=838)(C o n t i n u e d)$

\begin{tabular}{|c|c|c|c|c|c|c|c|c|c|c|c|c|c|}
\hline & \multirow{2}{*}{\multicolumn{2}{|c|}{ Overall ( $n=838)$}} & \multicolumn{11}{|c|}{ Hospitals } \\
\hline & & & \multicolumn{2}{|c|}{ AKU $(n=350,42 \%)$} & \multicolumn{2}{|c|}{ Kijabe $(n=105,13 \%)$} & \multicolumn{2}{|c|}{$\begin{array}{l}\text { Nyeri } \\
(n=110, \\
13 \%)\end{array}$} & \multicolumn{2}{|c|}{$\begin{array}{l}\text { St } \\
\text { Mary's } \\
(n=122, \\
15 \%)\end{array}$} & \multicolumn{2}{|c|}{$\begin{array}{l}\text { Others } \\
(n=151, \\
18 \%)\end{array}$} & \multirow[b]{2}{*}{$\mathrm{P}^{*}$} \\
\hline & $\mathrm{N}$ & $\%$ & $\mathrm{~N}$ & $\%$ & $\mathrm{~N}$ & $\%$ & $\mathrm{~N}$ & $\%$ & $\mathrm{~N}$ & $\%$ & $\mathrm{~N}$ & $\%$ & \\
\hline \multicolumn{14}{|l|}{ Reproductive } \\
\hline \multicolumn{14}{|l|}{ Age at menarche/year } \\
\hline $9-12$ & 68 & 8.5 & 30 & 8.6 & 10 & 11.1 & 7 & 6.9 & 7 & 5.8 & 14 & 10.0 & 0.40 \\
\hline $13-14$ & 340 & 42.4 & 150 & 42.9 & 31 & 34.4 & 39 & 38.2 & 61 & 50.8 & 59 & 42.1 & \\
\hline $15-20$ & 394 & 49.1 & 170 & 48.6 & 49 & 54.4 & 56 & 54.9 & 52 & 43.3 & 67 & 47.9 & \\
\hline Missing & 36 & & 0 & & 15 & & 8 & & 2 & & 11 & & \\
\hline \multicolumn{14}{|l|}{ Age at first pregnancy/year } \\
\hline Nulliparous $\neq$ & 37 & 4.5 & 23 & 6.6 & 4 & 3.9 & 2 & 1.9 & 2 & 1.8 & 6 & 4.0 & $<0.0001$ \\
\hline$<20$ & 216 & 26.3 & 62 & 17.7 & 29 & 28.4 & 34 & 31.8 & 38 & 34.2 & 53 & 35.3 & \\
\hline $20-24$ & 361 & 44.0 & 142 & 40.6 & 41 & 40.2 & 54 & 50.5 & 52 & 46.8 & 72 & 48.0 & \\
\hline $25-29$ & 150 & 18.3 & 90 & 25.7 & 21 & 20.6 & 14 & 13.1 & 14 & 12.6 & 11 & 7.3 & \\
\hline$\geq 30$ & 56 & 6.8 & 33 & 9.4 & 7 & 6.9 & 3 & 2.8 & 5 & 4.5 & 8 & 5.3 & \\
\hline Missing & 18 & & 0 & & 3 & & 3 & & 11 & & 1 & & \\
\hline \multicolumn{14}{|l|}{ Number of children } \\
\hline Nulliparous $\neq$ & 44 & 5.3 & 26 & 7.4 & 6 & 5.7 & 2 & 1.8 & 2 & 1.6 & 8 & 5.3 & 0.0001 \\
\hline 1 or 2 & 226 & 27.0 & 110 & 31.4 & 25 & 23.8 & 25 & 22.7 & 34 & 27.9 & 32 & 21.2 & \\
\hline 3 or 4 & 307 & 36.6 & 135 & 38.6 & 43 & 41.0 & 47 & 42.7 & 34 & 27.9 & 48 & 31.8 & \\
\hline$\geq 5$ & 261 & 31.2 & 79 & 22.6 & 31 & 29.5 & 36 & 32.7 & 52 & 42.6 & 63 & 41.7 & \\
\hline \multicolumn{14}{|l|}{ Cumulative breastfeeding duration/month } \\
\hline Nulliparousł & 37 & 4.6 & 23 & 6.6 & 4 & 4.0 & 2 & 1.9 & 2 & 1.7 & 6 & 4.2 & $0.013^{\mathrm{a}}$ \\
\hline Never breastfed & 7 & 0.9 & 3 & 0.9 & 2 & 2.0 & 0 & 0.0 & 0 & 0.0 & 2 & 1.4 & \\
\hline Q1: 1-<39 & 191 & 23.5 & 82 & 23.7 & 27 & 27.0 & 23 & 21.3 & 32 & 27.8 & 27 & 18.9 & \\
\hline Q2: 39-<62 & 192 & 23.6 & 100 & 28.9 & 28 & 28.0 & 24 & 22.2 & 16 & 13.9 & 24 & 16.8 & \\
\hline Q3: 62-<96 & 182 & 22.4 & 80 & 23.1 & 17 & 17.0 & 28 & 25.9 & 24 & 20.9 & 33 & 23.1 & \\
\hline Q4: $\geq 96$ & 203 & 25.0 & 58 & 16.8 & 22 & 22.0 & 31 & 28.7 & 41 & 35.7 & 51 & 35.7 & \\
\hline Missing & 26 & & 4 & & 5 & & 2 & & 7 & & 8 & & \\
\hline \multicolumn{14}{|c|}{ Averaged breastfeeding duration per child/month } \\
\hline Nulliparous $\neq$ & 37 & 4.6 & 23 & 6.6 & 4 & 4.0 & 2 & 1.9 & 2 & 1.7 & 6 & 4.2 & $0.0036^{\mathrm{a}}$ \\
\hline Never breastfed & 7 & 0.9 & 3 & 0.9 & 2 & 2.0 & 0 & 0.0 & 0 & 0.0 & 2 & 1.4 & \\
\hline$<12$ & 120 & 14.8 & 53 & 15.3 & 23 & 23.0 & 8 & 7.4 & 18 & 15.7 & 18 & 12.6 & \\
\hline $12-23$ & 407 & 50.1 & 153 & 44.2 & 46 & 46.0 & 71 & 65.7 & 64 & 55.7 & 73 & 51.0 & \\
\hline$\geq 24$ & 241 & 29.7 & 114 & 32.9 & 25 & 25.0 & 27 & 25.0 & 31 & 27.0 & 44 & 30.8 & \\
\hline Missing & 26 & & 4 & & 5 & & 2 & & 7 & & 8 & & \\
\hline \multicolumn{14}{|c|}{ Number of children and cumulative breastfeeding duration } \\
\hline Nulliparous or $\leq 3$ children and $<62$ months & 354 & 43.60 & 179 & 51.7 & 44 & 44.0 & 43 & 39.8 & 44 & 38.3 & 44 & 30.8 & $<0.0001$ \\
\hline$\leq 3$ children and $\geq 62$ months & 79 & 9.73 & 43 & 12.4 & 5 & 5.0 & 10 & 9.3 & 8 & 7.0 & 13 & 9.1 & \\
\hline$\geq 4$ children and $<62$ months & 73 & 8.99 & 29 & 8.4 & 17 & 17.0 & 6 & 5.6 & 6 & 5.2 & 15 & 10.5 & \\
\hline$\geq 4$ children and $\geq 62$ months & 306 & 37.69 & 95 & 27.5 & 34 & 34.0 & 49 & 45.4 & 57 & 49.6 & 71 & 49.7 & \\
\hline Missing & 26 & & 4 & & 5 & & 2 & & 7 & & 8 & & \\
\hline \multicolumn{14}{|l|}{ Age at first pregnancy and number of children } \\
\hline Nulliparous & 44 & 5.36 & 26 & 7.4 & 6 & 5.8 & 2 & 1.9 & 2 & 1.8 & 8 & 5.3 & $<0.0001$ \\
\hline Age $25+$ years, $1-3$ births & 154 & 18.76 & 98 & 28.0 & 16 & 15.5 & 13 & 12.1 & 13 & 11.7 & 14 & 9.3 & \\
\hline Age $<25$ years, $1-3$ births & 244 & 29.72 & 101 & 28.9 & 27 & 26.2 & 38 & 35.5 & 37 & 33.3 & 41 & 27.3 & \\
\hline Age $25+$ years, $4+$ births & 49 & 5.97 & 23 & 6.6 & 12 & 11.7 & 4 & 3.7 & 6 & 5.4 & 4 & 2.7 & \\
\hline
\end{tabular}


Table 1 Distributions of breast cancer risk factors in Kenyan breast cancer patients, overall and by hospitals (N=838) (Continued)

\begin{tabular}{|c|c|c|c|c|c|c|c|c|c|c|c|c|c|}
\hline & \multirow{2}{*}{\multicolumn{2}{|c|}{ Overall ( $n=838)$}} & \multicolumn{11}{|c|}{ Hospitals } \\
\hline & & & \multicolumn{2}{|c|}{ AKU $(n=350,42 \%)$} & \multicolumn{2}{|c|}{ Kijabe ( $n=105,13 \%)$} & \multicolumn{2}{|c|}{$\begin{array}{l}\text { Nyeri } \\
(n=110, \\
13 \%)\end{array}$} & \multicolumn{2}{|c|}{$\begin{array}{l}\text { St } \\
\text { Mary's } \\
(n=122, \\
15 \%)\end{array}$} & \multicolumn{2}{|c|}{$\begin{array}{l}\text { Others } \\
(n=151, \\
18 \%)\end{array}$} & \multirow[b]{2}{*}{$\mathrm{P}^{*}$} \\
\hline & $\mathrm{N}$ & $\%$ & $\mathrm{~N}$ & $\%$ & $\mathrm{~N}$ & $\%$ & $\mathrm{~N}$ & $\%$ & $\bar{N}$ & $\%$ & $\mathrm{~N}$ & $\%$ & \\
\hline Age $<25$ years, $4+$ births & 330 & 40.20 & 102 & 29.1 & 42 & 40.8 & 50 & 46.7 & 53 & 47.7 & 83 & 55.3 & \\
\hline Missing & 17 & & 0 & & 2 & & 3 & & 11 & & 1 & & \\
\hline \multicolumn{14}{|l|}{ Menopausal status } \\
\hline Premenopausal & 438 & 52.4 & 183 & 52.3 & 64 & 61.0 & 50 & 45.5 & 67 & 55.4 & 74 & 49.3 & 0.18 \\
\hline Postmenopausal & 398 & 47.6 & 167 & 47.7 & 41 & 39.0 & 60 & 54.5 & 54 & 44.6 & 76 & 50.7 & \\
\hline Missing & 2 & & 0 & & 0 & & 0 & & 1 & & 1 & & \\
\hline \multicolumn{14}{|l|}{ Age at menopause/year ${ }^{b}$} \\
\hline$<50$ & 191 & 57.4 & 91 & 55.8 & 18 & 62.1 & 28 & 59.6 & 20 & 71.4 & 34 & 51.5 & $0.45^{\mathrm{b}}$ \\
\hline$\geq 50$ & 142 & 42.6 & 72 & 44.2 & 11 & 37.9 & 19 & 40.4 & 8 & 28.6 & 32 & 48.5 & \\
\hline Missing & 65 & & 4 & & 12 & & 13 & & 26 & & 10 & & \\
\hline \multicolumn{14}{|c|}{ Cumulative hormonal contraception exposure/month } \\
\hline$<48$ & 216 & 45.9 & 88 & 46.3 & 25 & 44.6 & 26 & 40.0 & 27 & 34.6 & 50 & 61.0 & 0.014 \\
\hline 48 & 255 & 54.1 & 102 & 53.7 & 31 & 55.4 & 39 & 60.0 & 51 & 65.4 & 32 & 39.0 & \\
\hline Missing & 367 & & 160 & & 49 & & 45 & & 44 & & 69 & & \\
\hline
\end{tabular}

${ }^{*} P$ values were computed from chi-square tests except where noted. $P$ values less than 0.05 are shown in bold font. ${ }^{a}$ Nulliparous women and parous women who never breastfed were grouped together in chi-square test. ${ }^{b} \mathrm{Chi}$-square test was performed restricted to postmenopausal women. + Only $3.58 \%$ ( $n=30$ ) of study participants reported ever having smoked or used smokeless tobacco. Exposure to smoking is summarized here as exposed/never exposed, where exposed is defined as personal use of tobacco as well as exposure to smoke at the workplace or home during child or adulthood. $\neq$ Nulliparous cases were women who reported never pregnant, never given birth, and had no children ( $N=37,4.4 \%)$. AKU, Aga Khan University; BMI, body mass index; IQR, interquartile range; $\mathrm{Q}$, quartile; SD, standard deviation.

patients (Supplementary Figure 1), among whom obesity was significantly more prevalent than patients in other hospitals; however, this pattern was also observed among patients at Kijabe Hospital.

We further evaluated the associations between the risk factors and ER in younger ( $<50$ years) and older $(\geq 50$ years) women separately. In general, the associations with most risk factors were similar in younger and older women, except that we observed an association between older age at menarche and ER-negative patients in older $(\mathrm{OR}=2.25,95 \% \mathrm{CI}=1.04,4.84, \mathrm{P}=0.038$, comparing $\geq 15$ to $\leq 13$ years) but not in younger women $(\mathrm{OR}=0.98$, $95 \% \mathrm{CI}=0.52,1.87, \mathrm{P}=0.96$, comparing $\geq 15$ to $\leq 13$ years) (Supplementary Table 5).

\section{Associations between breast cancer risk factors and molecular subtypes}

Table 4 shows that the associations between $\mathrm{BC}$ risk factors and molecular subtypes defined by joint receptor status. Compared to luminal A patients, luminal B patients (combining luminal B-HER2+ and luminal B-high proliferative) were more likely to have lower parity (patients with 3 or 4 children, $\mathrm{OR}=0.47,95 \% \mathrm{CI}=0.28$, $0.79, p=0.005$; with 5 or more children, $\mathrm{OR}=0.45,95 \%$ $\mathrm{CI}=0.23,0.87, \mathrm{p}=0.018$, comparing to patients with 1 or 2 children). HER2-enriched patients were less likely to be obese $(\mathrm{OR}=0.36,95 \% \mathrm{CI}=0.16,0.81, p=0.013$, comparing $\geq 30$ to $<25 \mathrm{~kg} / \mathrm{m}^{2}$ ) or to have older age at menopause $(\mathrm{OR}=0.38,95 \% \mathrm{CI}=0.15,0.997, p=0.049$, comparing $\geq 50$ to $<50$ years). The HER2-BMI association appeared to be stronger among postmenopausal women $(\mathrm{OR}=0.24,95 \% \mathrm{CI}=0.07,0.081, p=$ 0.022) than among premenopausal women. Overall, cumulative or average breastfeeding duration did not vary significantly across subtypes. When looking at a number of children and breastfeeding or age at first birth jointly, it appears that luminal B patients with four or more children seemed to have shorter cumulative breastfeeding duration and later age at birth compared with luminal A patients (Table 4). Further stratifying luminal $\mathrm{B}$ and $\mathrm{TN}$ subtypes did not reveal additional associations (Supplementary Table 6).

We also conducted a number of sensitivity analyses to evaluate the impact of using grade to define subtypes when ki67 was missing and removing nulliparous women from analyses of age at first birth on our main conclusions. Overall, the results were similar to those from the original analyses (Supplementary Tables 7, 8, 9).

\section{Discussion}

The etiology of early-onset breast cancers is particularly lacking across populations given their rarity. 
Table 2 Distributions of tumor characteristics in Kenyan breast cancer patients, overall and by hospitals ( $N=838$ )

\begin{tabular}{|c|c|c|c|c|c|c|c|c|c|c|c|c|c|}
\hline \multirow[t]{3}{*}{ Tumor characteristic } & \multirow{2}{*}{\multicolumn{2}{|c|}{$\begin{array}{l}\text { Overall } \\
(n=838)\end{array}$}} & \multicolumn{11}{|c|}{ Hospitals } \\
\hline & & & \multicolumn{2}{|c|}{$\begin{array}{l}\text { AKU }(n=350, \\
42 \%)\end{array}$} & \multicolumn{2}{|c|}{$\begin{array}{l}\text { Kijabe }(n=105, \\
13 \%)\end{array}$} & \multicolumn{2}{|c|}{$\begin{array}{l}\text { Nyeri }(n=110, \\
13 \%)\end{array}$} & \multicolumn{2}{|c|}{$\begin{array}{l}\text { St Mary's ( } n= \\
122,15 \%)\end{array}$} & \multicolumn{2}{|c|}{$\begin{array}{l}\text { Others }(n=151 \text {, } \\
18 \%)\end{array}$} & \multirow[b]{2}{*}{$\mathrm{P}^{*}$} \\
\hline & $\mathbf{N}$ & $\%$ & $\mathrm{~N}$ & $\%$ & $\mathbf{N}$ & $\%$ & $\mathrm{~N}$ & $\%$ & $\mathrm{~N}$ & $\%$ & $\mathrm{~N}$ & $\%$ & \\
\hline \multicolumn{14}{|l|}{ Tumor subtypes } \\
\hline \multicolumn{14}{|l|}{ ER status } \\
\hline Negative & 256 & 30.6 & 101 & 28.9 & 25 & 23.8 & 45 & 40.9 & 32 & 26.2 & 53 & 35.1 & 0.029 \\
\hline Positive & 582 & 69.5 & 249 & 71.1 & 80 & 76.2 & 65 & 59.1 & 90 & 73.8 & 98 & 64.9 & \\
\hline \multicolumn{14}{|l|}{ PR status } \\
\hline Negative & 340 & 40.6 & 138 & 39.4 & 36 & 34.3 & 50 & 45.5 & 48 & 39.3 & 68 & 45.0 & 0.36 \\
\hline Positive & 498 & 59.4 & 212 & 60.6 & 69 & 65.7 & 60 & 54.5 & 74 & 60.7 & 83 & 55.0 & \\
\hline \multicolumn{14}{|l|}{ HER2 status } \\
\hline Negative & 596 & 72.6 & 246 & 71.3 & 75 & 72.1 & 81 & 76.4 & 91 & 74.6 & 103 & 71.5 & 0.84 \\
\hline Positive & 225 & 27.4 & 99 & 28.7 & 29 & 27.9 & 25 & 23.6 & 31 & 25.4 & 41 & 28.5 & \\
\hline Missing & 17 & & 5 & & 1 & & 4 & & 0 & & 7 & & \\
\hline \multicolumn{14}{|l|}{ Tumor molecular subtype } \\
\hline Luminal $A^{\mathbf{a}}$ & 286 & 34.8 & 121 & 35.1 & 46 & 44.2 & 37 & 34.9 & 40 & 32.8 & 42 & 29.2 & 0.080 \\
\hline Luminal B & 294 & 35.8 & 127 & 36.8 & 33 & 31.7 & 26 & 24.5 & 52 & 42.6 & 56 & 38.9 & \\
\hline HER2-enriched & 88 & 10.7 & 37 & 10.7 & 11 & 10.6 & 14 & 13.2 & 12 & 9.8 & 14 & 9.7 & \\
\hline Triple negative & 153 & 18.6 & 60 & 17.4 & 14 & 13.5 & 29 & 27.4 & 18 & 14.8 & 32 & 22.2 & \\
\hline Missing & 17 & & 5 & & 1 & & 4 & & 0 & & 7 & & \\
\hline Luminal $A^{\mathbf{a}}$ & 286 & 36.9 & 121 & 36.7 & 46 & 48.9 & 37 & 37.4 & 40 & 33.9 & 42 & 31.1 & 0.094 \\
\hline Luminal B - HER2- & 157 & 20.2 & 65 & 19.7 & 15 & 16.0 & 15 & 15.2 & 33 & 28.0 & 29 & 21.5 & \\
\hline Luminal B - HER2 ${ }^{+}$ & 137 & 17.7 & 62 & 18.8 & 18 & 19.1 & 11 & 11.1 & 19 & 16.1 & 27 & 20.0 & \\
\hline HER2-enriched & 88 & 11.3 & 37 & 11.2 & 11 & 11.7 & 14 & 14.1 & 12 & 10.2 & 14 & 10.4 & \\
\hline Core-basal like & 57 & 7.3 & 24 & 7.3 & 2 & 2.1 & 13 & 13.1 & 7 & 5.9 & 11 & 8.1 & \\
\hline Five negative & 51 & 6.6 & 20 & 6.4 & 2 & 2.1 & 9 & 9.1 & 7 & 5.9 & 12 & 8.9 & \\
\hline Missing & $62^{\mathrm{b}}$ & & 20 & & 11 & & 11 & & 4 & & 16 & & \\
\hline \multicolumn{14}{|l|}{ Tumor pathology } \\
\hline \multicolumn{14}{|l|}{ Surgery } \\
\hline Core biopsy only & 435 & 51.9 & 157 & 44.9 & 26 & 24.8 & 46 & 41.8 & 73 & 59.8 & 133 & 88.1 & $<0.0001$ \\
\hline Lumpectomy or mastectomy & 403 & 48.1 & 193 & 55.1 & 79 & 75.2 & 64 & 58.2 & 49 & 40.2 & 18 & 11.9 & \\
\hline \multicolumn{14}{|l|}{ Tumor size $(\mathrm{cm})$} \\
\hline$<2$ & 41 & 7.6 & 29 & 13.1 & 8 & 8.1 & 2 & 2.3 & 1 & 1.9 & 1 & 1.3 & $<0.0001$ \\
\hline $2-<5$ & 287 & 53.5 & 134 & 60.4 & 53 & 53.5 & 39 & 44.8 & 26 & 50.0 & 35 & 45.5 & \\
\hline$\geq 5$ & 209 & 38.9 & 59 & 26.6 & 38 & 38.4 & 46 & 52.9 & 25 & 48.1 & 41 & 53.3 & \\
\hline Missing $^{c}$ & 301 & & 128 & & 6 & & 23 & & 70 & & 74 & & \\
\hline \multicolumn{14}{|l|}{ Tumor overall grade } \\
\hline Grade 1 (low) & 35 & 5.0 & 20 & 7.3 & 4 & 4.4 & 6 & 5.7 & 2 & 1.7 & 3 & 2.8 & 0.17 \\
\hline Grade 2 (intermediate) & 319 & 45.9 & 130 & 47.3 & 34 & 37.8 & 44 & 41.5 & 59 & 50.9 & 52 & 48.2 & \\
\hline Grade 3 (high) & 341 & 49.1 & 125 & 45.5 & 52 & 57.8 & 56 & 52.8 & 55 & 47.4 & 53 & 49.1 & \\
\hline Missing/not applicable & 143 & & 75 & & 15 & & 4 & & 6 & & 43 & & \\
\hline \multicolumn{14}{|l|}{ Lymphovascular invasion } \\
\hline No & 326 & 38.9 & 139 & 39.7 & 30 & 28.6 & 34 & 30.9 & 47 & 38.5 & 76 & 50.3 & 0.0029 \\
\hline Yes & 512 & 61.1 & 211 & 60.3 & 75 & 71.4 & 76 & 69.1 & 75 & 61.5 & 75 & 49.7 & \\
\hline
\end{tabular}

Among cases with lumpectomy or mastectomy $(n=403)$ : 
Table 2 Distributions of tumor characteristics in Kenyan breast cancer patients, overall and by hospitals ( $N=838)$ (Continued)

\begin{tabular}{|c|c|c|c|c|c|c|c|c|c|c|c|c|c|}
\hline \multirow[t]{3}{*}{ Tumor characteristic } & \multirow{2}{*}{\multicolumn{2}{|c|}{$\begin{array}{l}\text { Overall } \\
(n=838)\end{array}$}} & \multicolumn{11}{|c|}{ Hospitals } \\
\hline & & & \multicolumn{2}{|c|}{$\begin{array}{l}\text { AKU }(n=350, \\
42 \%)\end{array}$} & \multicolumn{2}{|c|}{$\begin{array}{l}\text { Kijabe }(n=105 \text {, } \\
13 \%)\end{array}$} & \multicolumn{2}{|c|}{$\begin{array}{l}\text { Nyeri }(n=110 \text {, } \\
13 \%)\end{array}$} & \multicolumn{2}{|c|}{$\begin{array}{l}\text { St Mary's ( } n= \\
122,15 \%)\end{array}$} & \multicolumn{2}{|c|}{$\begin{array}{l}\text { Others }(n=151, \\
18 \%)\end{array}$} & \multirow[b]{2}{*}{$\mathrm{P}^{*}$} \\
\hline & $\mathbf{N}$ & $\%$ & $\mathbf{N}$ & $\%$ & $\bar{N}$ & $\%$ & $\mathrm{~N}$ & $\%$ & $\bar{N}$ & $\%$ & $\bar{N}$ & $\%$ & \\
\hline \multicolumn{14}{|l|}{ Tumor stage } \\
\hline Stage $0, \mathrm{i}$ & 30 & 8.2 & 21 & 12.1 & 2 & 2.6 & 6 & 10.3 & 1 & 2.4 & 0 & 0.0 & 0.0040 \\
\hline Stage ii & 154 & 42.3 & 85 & 49.1 & 30 & 39.5 & 20 & 34.5 & 12 & 29.3 & 7 & 43.8 & \\
\hline Stage iii, iv & 180 & 49.5 & 67 & 38.7 & 44 & 57.9 & 32 & 55.2 & 28 & 68.3 & 9 & 56.3 & \\
\hline Missing & 39 & & 20 & & 3 & & 6 & & 8 & & 2 & & \\
\hline \multicolumn{14}{|c|}{ Lymph nodes with metastasis } \\
\hline No & 162 & 40.2 & 89 & 46.1 & 23 & 29.1 & 22 & 34.4 & 20 & 40.8 & 8 & 44.4 & 0.96 \\
\hline Yes & 241 & 59.8 & 104 & 53.9 & 56 & 70.9 & 42 & 65.6 & 29 & 59.2 & 10 & 55.6 & \\
\hline \multicolumn{14}{|l|}{ Extranodal extension } \\
\hline No & 244 & 60.6 & 132 & 68.4 & 39 & 49.4 & 35 & 54.7 & 26 & 53.1 & 12 & 66.7 & 0.022 \\
\hline Yes & 159 & 39.5 & 61 & 31.6 & 40 & 50.6 & 29 & 45.3 & 23 & 46.9 & 6 & 33.3 & \\
\hline
\end{tabular}

* $P$ values were computed from chi-square test except where noted. $P$ values less than 0.05 are shown in bold font. ${ }^{\text {a }}$ Seventy-four cases, who had missing data for both ki67 and tumor grade, were grouped into the subcategory "Luminal A" in tumor molecular subtype. "'Sixty-two cases whose tumor molecular subtype

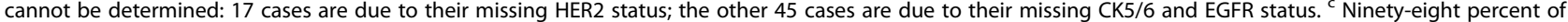
missingness are from cases with core biopsy only. AKU, Aga Khan University; CK5/6, cytokeratin 5/6; EGFR, epidermal growth factor receptor; ER, estrogen receptor; HER2, human epidermal growth factor receptor-2; PR, progesterone receptor

Studying African populations where risk factors differ and where onset is almost a decade earlier could provide new insights on breast cancer etiology given the etiologic and molecular subtype heterogeneity in diverse populations.

There is limited data from Africa where some of the breast cancer-associated risk/protective factors such as parity and breastfeeding have extremely different distributions. The overall risk factor distribution for $\mathrm{BC}$ patients in our study is similar to a large case-control study from Ghana [19], but is strikingly different from that of other populations including African Americans [20-22]. As an example, among BC patients in Ghana and Kenya, $>60 \%$ of women had $\geq 3$ children, $>80 \%$ women had the first child before age 25 years, and > $90 \%$ women had breastfed with the average breastfeeding duration per child near two years. Whereas among African American $\mathrm{BC}$ patients in the African American Breast Cancer Epidemiology and Risk (AMBER) consortium, only $35 \%$ had $\geq 3$ children and $>40 \%$ had never breastfed [21]. Similarly, the prevalence of obesity (BMI $>30 \mathrm{~kg} / \mathrm{m}^{2}, 41.7 \%$ in AMBER vs. $29.4 \%$ in Kenya) and early age at menarche $(<13$ years, $52.3 \%$ in AMBER vs. $8.5 \%$ in Kenya) was much higher in AMBER [22, 23] than in Kenya. On the other hand, the frequency of ER-negative cancers (AMBER: 33.9\%; Kenya: 30.5\%) and TNBC (AMBER: 15.3\%; Kenya: 18.6\%) was similar in AMBER and Kenya, which is lower compared to BC patients in Ghana (ER-: 50\%; TNBC: 28\%).
Parity has been reported to have a dual effect on breast cancer risk; it is protective for ER+ women while increases risk for ER- women especially among younger women $[24,21]$. Despite the heterogeneity in parityrelated exposures, the differential effect of parity by ER has been consistently reported across different populations $[25,21,19,26]$. Although we were not able to compare relative risks associated with parity in different molecular subtypes due to the case-only design, our results of higher parity in ER-negative than in ER-positive patients is consistent with results from previous casecontrol studies $[19,26]$. In particular, taking advantage of the much higher parity among patients in Kenya, we observed that the association of parity with ER followed a dose-dependent manner, with the highest variation by ER observed among women with five or more children. Similarly, in a population where the vast majority of women had their first children before the age of 30 years, we found a similar association between younger age at first birth and ER-negative breast cancer consistent with previous studies [27, 26, 28], supporting increased parity as a risk factor for ER-negative breast cancers across multiple populations. We observed luminal B patients, both luminal $\mathrm{B} /$ high proliferative and luminal B/HER2+, had fewer children compared to luminal A patients. These results are in line with data from the Nurse's Health Study reporting greater reduced risks associated with parity in luminal B than luminal A patients [25], suggesting that parity may have a stronger protective effect for luminal B as compared to luminal A patients. However, using data based on a Malaysian case-series, 
Table 3. Associations between breast cancer risk factors and ER status in Kenyan breast cancer patients $(N=838)$

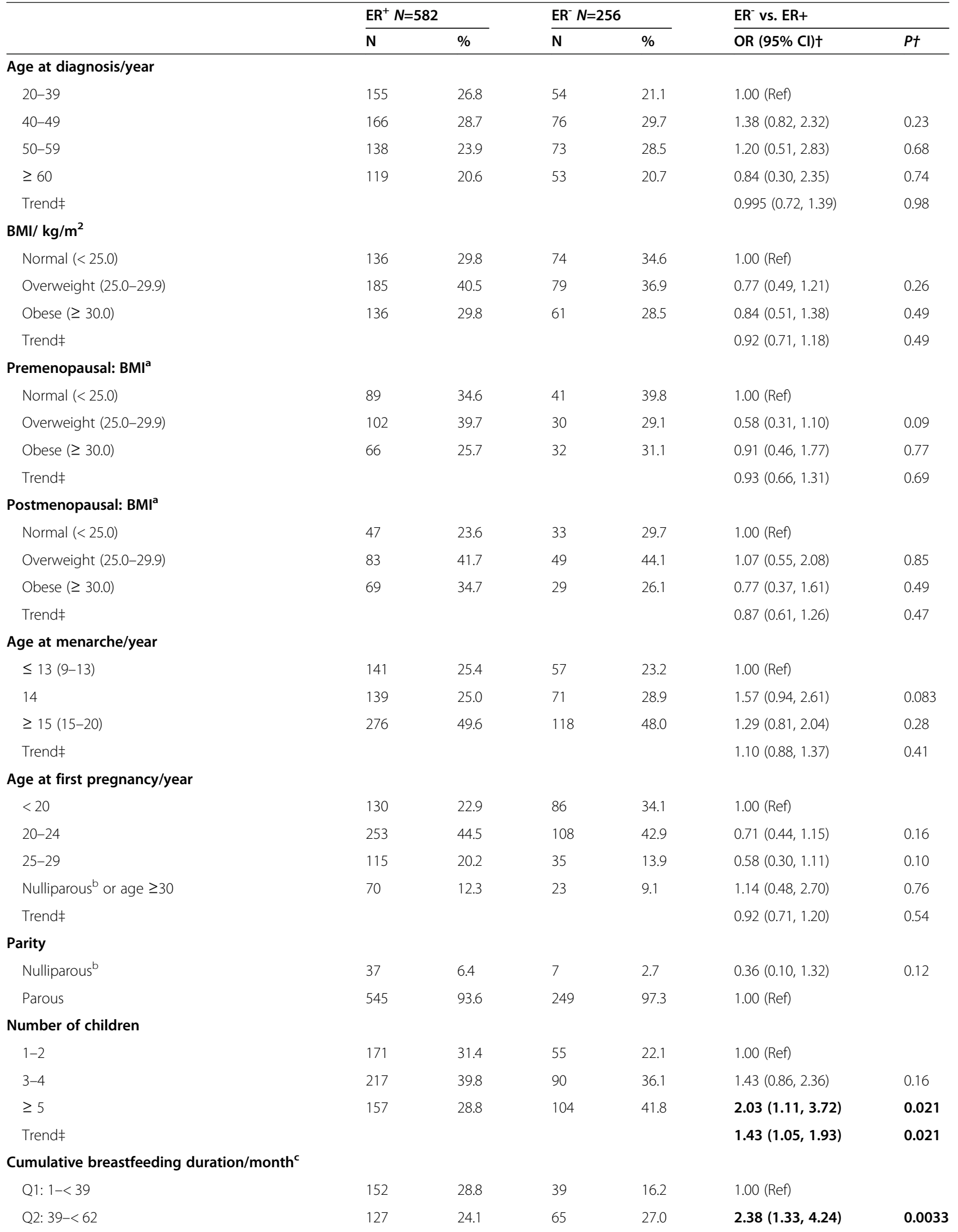


Table 3. Associations between breast cancer risk factors and ER status in Kenyan breast cancer patients ( $N=838$ ) (Continued)

\begin{tabular}{|c|c|c|c|c|c|c|}
\hline & \multicolumn{2}{|c|}{$\mathrm{ER}^{+} \mathrm{N}=582$} & \multicolumn{2}{|c|}{$\mathrm{ER}^{-} \mathrm{N}=\mathbf{2 5 6}$} & \multicolumn{2}{|l|}{ ER $^{-}$vs. ER+ } \\
\hline & $\mathbf{N}$ & $\%$ & $\mathbf{N}$ & $\%$ & OR $(95 \% \mathrm{Cl}) \dagger$ & $P t$ \\
\hline Q3: 62-<96 & 124 & 23.5 & 58 & 24.1 & $1.44(0.74,2.80)$ & 0.28 \\
\hline Q4: $\geq 96$ & 124 & 23.5 & 79 & 32.8 & $1.58(0.76,3.30)$ & 0.22 \\
\hline Trend $\neq$ & & & & & $1.10(0.87,1.39)$ & 0.43 \\
\hline \multicolumn{7}{|l|}{ Mean breastfeeding duration per child/month } \\
\hline$<12$ & 84 & 15.9 & 36 & 14.9 & 1.00 (Ref) & \\
\hline $12-23$ & 277 & 52.6 & 130 & 53.9 & $1.10(0.62,1.94)$ & 0.74 \\
\hline$\geq 24$ & 166 & 31.5 & 75 & 31.1 & $1.25(0.68,2.30)$ & 0.48 \\
\hline Trend $\neq$ & & & & & $1.12(0.83,1.51)$ & 0.45 \\
\hline \multicolumn{7}{|l|}{ Age at first pregnancy and number of children } \\
\hline Age $25+$ years, $1-3$ births & 117 & 22.0 & 37 & 15.0 & 1.00 (Ref) & \\
\hline Age $<25$ years, $1-3$ births & 173 & 32.6 & 71 & 28.9 & $1.20(0.68,2.12)$ & 0.53 \\
\hline Age $25+$ years, $4+$ births & 34 & 6.4 & 15 & 6.1 & $1.47(0.60,3.61)$ & 0.40 \\
\hline Age $<25$ years, $4+$ births & 207 & 39.0 & 123 & 50.0 & $1.69(0.93,3.05)$ & 0.085 \\
\hline Trend $\neq$ & & & & & $1.19(0.99,1.43)$ & 0.063 \\
\hline \multicolumn{7}{|c|}{ Number of children and cumulative breastfeeding duration } \\
\hline Nulliparous or $\leq 3$ children and $<62$ months & 265 & 47.0 & 89 & 35.9 & 1.00 (Ref) & \\
\hline$\leq 3$ children and $\geq 62$ months & 56 & 9.9 & 23 & 9.3 & $1.09(0.56,2.09)$ & 0.81 \\
\hline$\geq 4$ children and $<62$ months & 51 & 9.0 & 22 & 8.9 & $1.52(0.74,3.09)$ & 0.25 \\
\hline$\geq 4$ children and $\geq 62$ months & 192 & 34.0 & 114 & 46.0 & $1.44(0.89,2.34)$ & 0.14 \\
\hline Trend $\neq$ & & & & & $1.14(0.97,1.33)$ & 0.12 \\
\hline \multicolumn{7}{|l|}{ Menopausal status $^{\mathrm{a}}$} \\
\hline Premenopausal & 317 & 54.6 & 121 & 47.5 & 1.00 (Ref) & \\
\hline Postmenopausal & 264 & 45.4 & 134 & 52.5 & $1.44(0.74,2.81)$ & 0.28 \\
\hline \multicolumn{7}{|l|}{ Age at menopause/year ${ }^{d}$} \\
\hline$<50$ & 117 & 21.8 & 74 & 31.5 & 1.00 (Ref) & \\
\hline$\geq 50$ & 102 & 19.0 & 40 & 17.0 & $0.73(0.40,1.36)$ & 0.32 \\
\hline
\end{tabular}

† Point estimates and 95\% confidence intervals were from multivariable models, adjusting for the same series of covariates (except where noticed): age at diagnosis, BMI, age at menarche, age at first pregnancy, number of children, mean breastfeeding duration per child, age at menopause, family history of breast cancer in first-degree female relative, occupation, education level, and location of facility. Estimates of numbers of children, cumulative and averaged breastfeeding duration, and combined age at first pregnancy and number of children were computed among parous women. $\neq$ Results were from the trend analysis using the categorical risk factor as a trend. ${ }^{a}$ Multivariable modeling analysis without adjusting for age at menopause. ${ }^{b}$ Women who reported never pregnant, never gave birth, and had no child were grouped as "Nulliparous" in modeling analyses. " Multivariable modeling analysis without adjusting for mean breastfeeding duration per child. ${ }^{\mathrm{d}}$ Multivariable Modeling analysis was restricted to postmenopausal women. BMI, body mass index; $\mathrm{Cl}$, confidence interval; ER, estrogen receptor; $\mathrm{OR}$, odds ratio; $\mathrm{Q}$, quartile

we found that luminal B patients were more likely to be parous and to have breastfed compared to luminal A patients [26]. These inconsistent results warrant further investigations especially in diverse populations.

Investigations of associations between breastfeeding and breast cancer risk by receptor status have resulted in inconsistent findings, with some showing a similar protective effect for all subtypes [29], and others showing a stronger protection against ER-negative especially TNBC [30]. In the Ghana study in which the frequency of ER-negative breast cancer especially TNBC was higher ( $28 \%$ vs $18 \%$ of tumors) than in the Kenya study, the increased risk of parity was offset by more extended breastfeeding, which was only seen among patients $<50$ years of age in ER-negative but not in ER-positive patients, while in older women, extended breastfeeding showed an inverse association regardless of ER status yet a stronger association for ER-positive patients [19]. We did not observe significant differences of breastfeeding by ER or by intrinsic subtype, either in all women or by age. The inconsistent findings between different African populations with similar parity and breastfeeding characteristics highlight the complexity of subtype-specific risk associations and the importance of conducting large molecular epidemiologic studies in diverse African populations. 


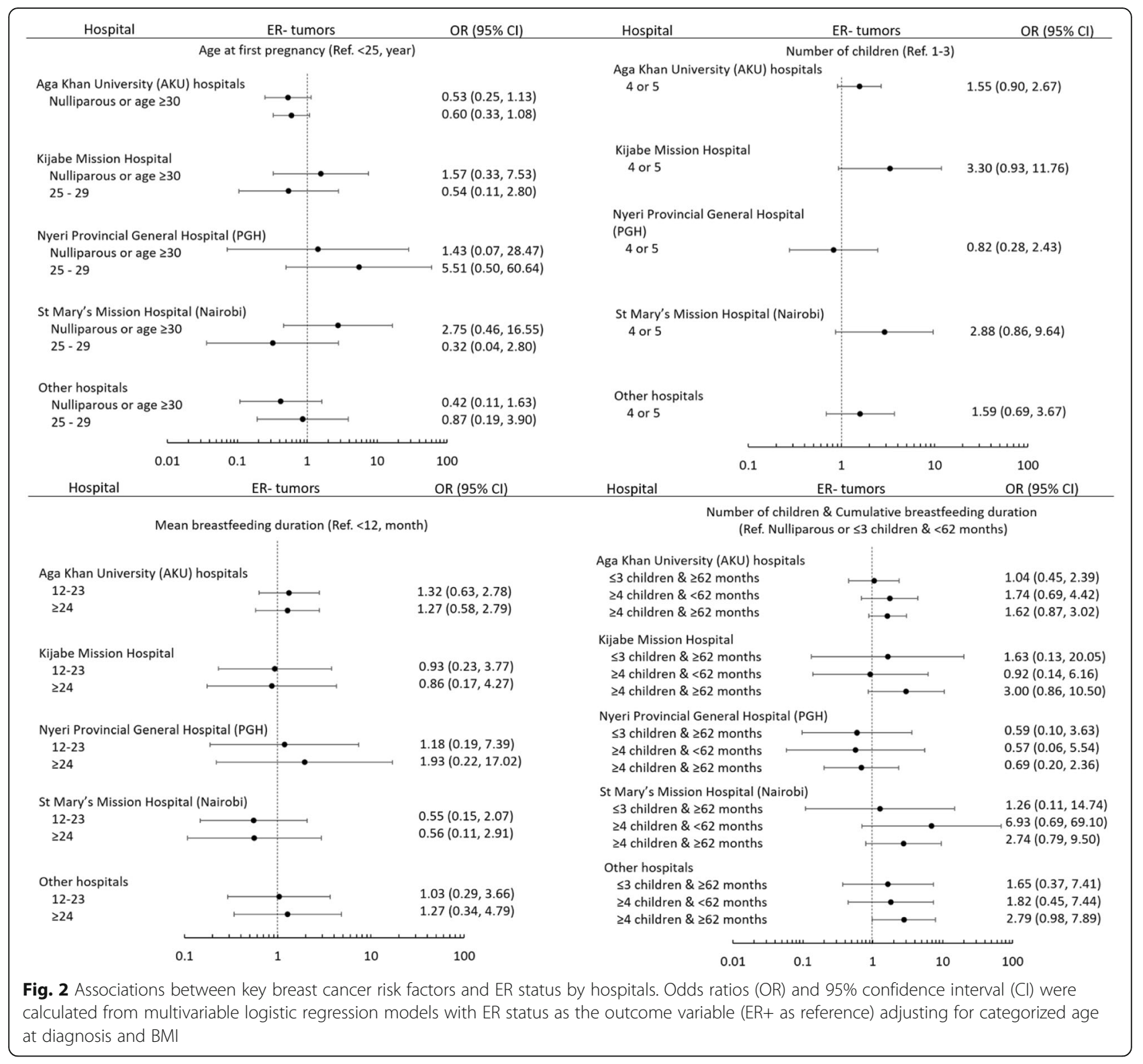

Obesity is a known risk factor for breast cancer in post-menopausal women but protective in premenopausal women [31]. Obesity can disrupt some biological pathways, resulting in insulin resistance, and synthesis of endogenous sex hormones [32,33]. When we examined the association of obesity with molecular subtypes, we found that patients with HER2 enriched $\mathrm{BC}$ were less likely to have a high BMI. Although we cannot completely rule out the possibility of reverse causality due to weight loss associated with breast cancer, it is unlikely that the association we observed is entirely driven by reverse causation since BMI did not vary significantly by tumor stage in our study. Our findings are consistent with a Polish breast cancer case-control study, which found that in premenopausal women, HER2 expression was inversely associated with BMI adjusted for the 4 markers (adjusted $p$-trend $=0.01$ ) [34]. In addition, the association was stronger among AKU patients, who were more likely to have early-stage disease as compared to patients from other hospitals. Our findings are similar to a study conducted in Malaysia, which showed that women with HER2-enriched and TNBC tumors were significantly less likely to be obese than those with the luminal A subtype [26]. Our results are also in line with the analysis based on African Americans in the AMBER consortium [22] and a pooled analysis of nine studies of the National Cancer Institute cohort consortium [27] showing that, among postmenopausal women, higher recent BMI was associated with increased risk of ERpositive cancer, but was either associated with decreased 
Table 4 Associations between breast cancer risk factors and tumor molecular subtypes in Kenyan breast cancer patients $\left(N=821^{*}\right)$

\begin{tabular}{|c|c|c|c|c|c|c|c|c|c|c|c|c|c|c|}
\hline & \multirow{2}{*}{\multicolumn{2}{|c|}{$\begin{array}{l}\text { Luminal } \\
\text { A } n=286\end{array}$}} & \multirow{2}{*}{\multicolumn{2}{|c|}{$\begin{array}{l}\text { Luminal } \\
\text { B } n=294\end{array}$}} & \multirow{2}{*}{\multicolumn{2}{|c|}{$\begin{array}{l}\text { Luminal B vs. } \\
\text { Luminal A }\end{array}$}} & \multicolumn{4}{|c|}{ Tumor subtypes } & \multirow{2}{*}{\multicolumn{2}{|c|}{$\begin{array}{l}\text { Triple } \\
\text { negative } \\
n=153\end{array}$}} & \multirow{2}{*}{\multicolumn{2}{|c|}{$\begin{array}{l}\text { Triple negative vs } \\
\text { Luminal A }\end{array}$}} \\
\hline & & & & & & & \multicolumn{2}{|c|}{$\begin{array}{l}\text { HER2- } \\
\text { enriched } \\
n=88\end{array}$} & \multicolumn{2}{|c|}{$\begin{array}{l}\text { HER2-enriched vs. } \\
\text { Luminal A }\end{array}$} & & & & \\
\hline & $\mathbf{N}$ & $\%$ & $\mathrm{~N}$ & $\%$ & $\begin{array}{l}\text { OR }(95 \% \\
\text { Cl)† }\end{array}$ & $P t$ & $\mathrm{~N}$ & $\%$ & $\begin{array}{l}\text { OR }(95 \% \\
\text { Cl)† }\end{array}$ & $P t$ & $\mathrm{~N}$ & $\%$ & $\begin{array}{l}\text { OR }(95 \% \\
\text { Cl)† }\end{array}$ & $P t$ \\
\hline \multicolumn{15}{|l|}{ Age at diagnosis/year } \\
\hline$<50$ & 142 & 50.2 & 180 & 61.4 & 1.00 (Ref) & & 44 & 50.0 & 1.00 (Ref) & & 77 & 50.3 & 1.00 (Ref) & \\
\hline$\geq 50$ & 141 & 49.8 & 113 & 38.6 & $\begin{array}{l}0.87(0.36 \\
2.06)\end{array}$ & 0.74 & 44 & 50.0 & $\begin{array}{l}1.84(0.57 \\
5.92)\end{array}$ & 0.30 & 76 & 49.7 & $\begin{array}{l}0.52(0.20 \\
1.40)\end{array}$ & 0.20 \\
\hline \multicolumn{15}{|l|}{$\mathrm{BMI} / \mathrm{kg} / \mathrm{m}^{2}$} \\
\hline Normal $(<25.0)$ & 60 & 27.3 & 71 & 30.5 & 1.00 (Ref) & & 29 & 41.4 & 1.00 (Ref) & & 43 & 32.6 & 1.00 (Ref) & \\
\hline Overweight (25.0-29.9) & 84 & 38.2 & 99 & 42.5 & $\begin{array}{l}1.09(0.66 \\
1.82)\end{array}$ & 0.73 & 25 & 35.7 & $\begin{array}{l}0.55(0.28 \\
1.11)\end{array}$ & 0.10 & 49 & 37.1 & $\begin{array}{l}0.91(0.49 \\
1.68)\end{array}$ & 0.75 \\
\hline Obese $(\geq 30.0)$ & 76 & 34.5 & 63 & 27.0 & $\begin{array}{l}0.76(0.43 \\
1.33)\end{array}$ & 0.33 & 16 & 22.9 & $\begin{array}{l}0.36(0.16, \\
0.81)\end{array}$ & 0.013 & 40 & 30.3 & $\begin{array}{l}0.89(0.46 \\
1.72)\end{array}$ & 0.73 \\
\hline Trend $\neq$ & & & & & $\begin{array}{l}0.87(0.66 \\
1.15)\end{array}$ & 0.32 & & & $\begin{array}{l}0.59(0.39 \\
0.88)\end{array}$ & 0.011 & & & $\begin{array}{l}0.94(0.68, \\
1.32)\end{array}$ & 0.73 \\
\hline \multicolumn{15}{|l|}{ Premenopausal: $\mathrm{BMI}^{\mathrm{a}}$} \\
\hline Normal $(<25.0)$ & 36 & 32.4 & 51 & 34.7 & 1.00 (Ref) & & 16 & 45.7 & 1.00 (Ref) & & 23 & 38.3 & 1.00 (Ref) & \\
\hline Overweight (25.0-29.9) & 43 & 38.7 & 59 & 40.1 & $\begin{array}{l}0.98(0.51 \\
1.88)\end{array}$ & 0.95 & 11 & 31.4 & $\begin{array}{l}0.52(0.19 \\
1.40)\end{array}$ & 0.20 & 16 & 26.7 & $\begin{array}{l}0.49(0.20 \\
1.21)\end{array}$ & 0.12 \\
\hline Obese $(\geq 30.0)$ & 32 & 28.8 & 37 & 25.2 & $\begin{array}{l}0.78(0.37 \\
1.61)\end{array}$ & 0.49 & 8 & 22.9 & $\begin{array}{l}0.44(0.14 \\
1.35)\end{array}$ & 0.15 & 21 & 35.0 & $\begin{array}{l}0.84(0.34 \\
2.07)\end{array}$ & 0.71 \\
\hline Trend $\neq$ & & & & & $\begin{array}{l}0.89(0.62 \\
1.27)\end{array}$ & 0.52 & & & $\begin{array}{l}0.65(0.37 \\
1.1)\end{array}$ & 0.13 & & & $\begin{array}{l}0.91(0.57 \\
1.45)\end{array}$ & 0.69 \\
\hline \multicolumn{15}{|l|}{ Postmenopausal: $\mathrm{BMI}^{\mathrm{a}}$} \\
\hline Normal $(<25.0)$ & 24 & 22.0 & 20 & 23.5 & 1.00 (Ref) & & 13 & 37.1 & 1.00 (Ref) & & 20 & 27.8 & 1.00 (Ref) & \\
\hline Overweight (25.0-29.9) & 41 & 37.6 & 40 & 47.1 & $\begin{array}{l}1.35(0.57 \\
3.20)\end{array}$ & 0.50 & 14 & 40.0 & $\begin{array}{l}0.48(0.17 \\
1.40)\end{array}$ & 0.18 & 33 & 45.8 & $\begin{array}{l}1.70(0.68 \\
4.26)\end{array}$ & 0.25 \\
\hline Obese $(\geq 30.0)$ & 44 & 40.4 & 25 & 29.4 & $\begin{array}{l}1.10(0.43 \\
2.82)\end{array}$ & 0.84 & 8 & 22.9 & $\begin{array}{l}0.24(0.07 \\
0.81)\end{array}$ & 0.022 & 19 & 26.4 & $\begin{array}{l}1.28(0.48 \\
3.46)\end{array}$ & 0.62 \\
\hline Trend $\neq$ & & & & & $\begin{array}{l}1.02(0.64 \\
1.64)\end{array}$ & 0.92 & & & $\begin{array}{l}0.47(0.26 \\
0.88)\end{array}$ & 0.018 & & & $\begin{array}{l}1.10(0.68, \\
1.78)\end{array}$ & 0.71 \\
\hline \multicolumn{15}{|l|}{ Age at menarche/year } \\
\hline$\leq 13(9-13)$ & 69 & 25.8 & 71 & 24.8 & 1.00 (Ref) & & 23 & 26.4 & 1.00 (Ref) & & 33 & 22.9 & 1.00 (Ref) & \\
\hline 14 & 68 & 25.4 & 69 & 24.1 & $\begin{array}{l}1.28(0.73 \\
2.25)\end{array}$ & 0.39 & 26 & 29.9 & $\begin{array}{l}1.65(0.73 \\
3.74)\end{array}$ & 0.23 & 37 & 25.7 & $\begin{array}{l}1.43(0.73 \\
2.81)\end{array}$ & 0.30 \\
\hline$\geq 15(15-20)$ & 131 & 48.9 & 146 & 51.1 & $\begin{array}{l}1.29(0.79 \\
2.13)\end{array}$ & 0.31 & 38 & 43.7 & $\begin{array}{l}1.32(0.64 \\
2.73)\end{array}$ & 0.46 & 74 & 51.4 & $\begin{array}{l}1.43(0.78 \\
2.60)\end{array}$ & 0.24 \\
\hline Trend $\neq$ & & & & & $\begin{array}{l}1.13(0.88 \\
1.45)\end{array}$ & 0.34 & & & $\begin{array}{l}1.12(0.78 \\
1.59)\end{array}$ & 0.54 & & & $\begin{array}{l}1.18(0.88, \\
1.58)\end{array}$ & 0.28 \\
\hline \multicolumn{15}{|c|}{ Age at first pregnancy/year } \\
\hline$<20$ & 72 & 26.2 & 58 & 19.9 & 1.00 (Ref) & & 31 & 35.6 & 1.00 (Ref) & & 51 & 34.0 & 1.00 (Ref) & \\
\hline $20-24$ & 121 & 44.0 & 133 & 45.5 & $\begin{array}{l}1.51(0.84 \\
2.70)\end{array}$ & 0.17 & 30 & 34.5 & $\begin{array}{l}0.64(0.29 \\
1.37)\end{array}$ & 0.25 & 69 & 46.0 & $\begin{array}{l}0.81(0.43 \\
1.51)\end{array}$ & 0.51 \\
\hline $25-29$ & 51 & 18.5 & 62 & 21.2 & $\begin{array}{l}1.76(0.85 \\
3.65)\end{array}$ & 0.13 & 16 & 18.4 & $\begin{array}{l}0.92(0.34 \\
2.51)\end{array}$ & 0.87 & 19 & 12.7 & $\begin{array}{l}0.67(0.28 \\
1.60)\end{array}$ & 0.37 \\
\hline Nulliparous $^{\mathrm{b}}$ or $\geq 30$ & 31 & 11.3 & 39 & 13.4 & $\begin{array}{l}1.06(0.40 \\
2.82)\end{array}$ & 0.90 & 10 & 11.5 & $\begin{array}{l}1.18(0.31 \\
4.53)\end{array}$ & 0.81 & 11 & 7.3 & $\begin{array}{l}0.66(0.20 \\
2.18)\end{array}$ & 0.50 \\
\hline Trend $\neq$ & & & & & $\begin{array}{l}1.09(0.82 \\
1.45)\end{array}$ & 0.54 & & & $\begin{array}{l}1.02(0.67 \\
1.56)\end{array}$ & 0.93 & & & $\begin{array}{l}0.84(0.59 \\
1.20)\end{array}$ & 0.33 \\
\hline
\end{tabular}


Table 4 Associations between breast cancer risk factors and tumor molecular subtypes in Kenyan breast cancer patients ( $N=821^{*}$ ) (Continued)

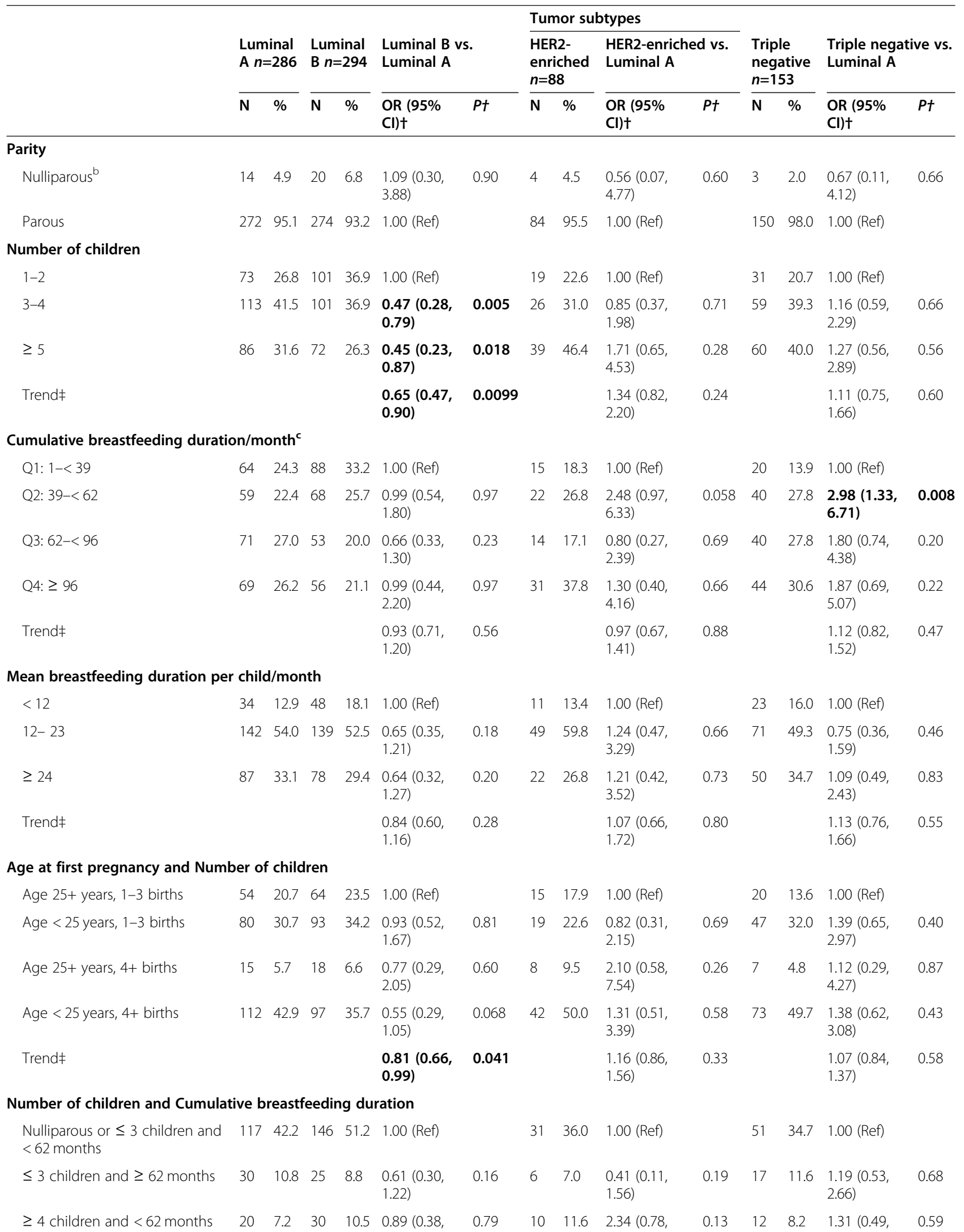


Table 4 Associations between breast cancer risk factors and tumor molecular subtypes in Kenyan breast cancer patients ( $N=821^{*}$ ) (Continued)

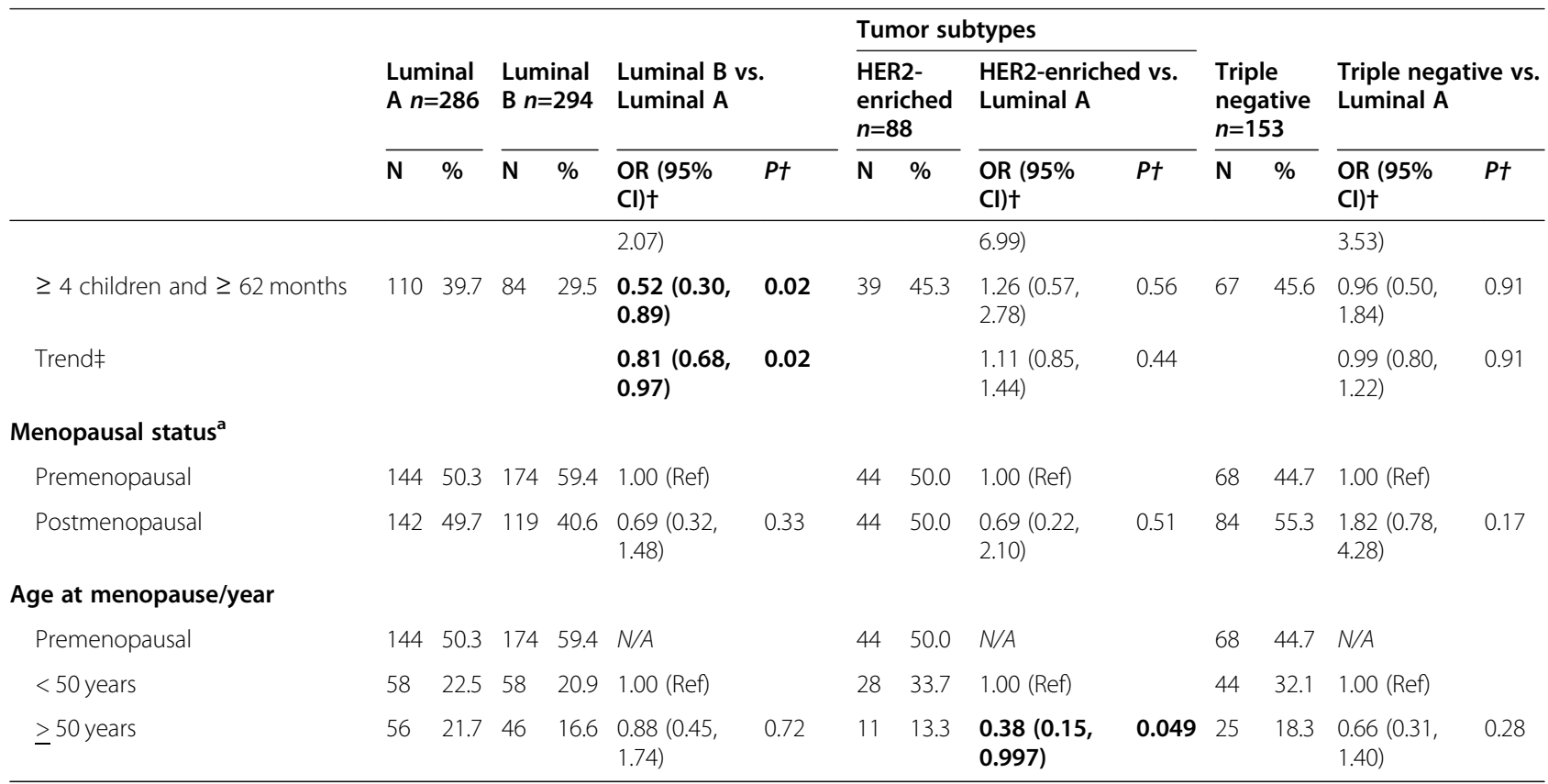

*Seventeen cases were excluded from analyses because of their missing data for HER2 status. † Point estimates and $95 \%$ confidence intervals were from multivariable models, adjusting for the same series of covariates (except where noticed): age at diagnosis, BMI, age at menarche, age at first pregnancy, number of children, mean breastfeeding duration per child, age at menopause, family history of breast cancer in first-degree female relative, occupation, education level, and location of the facility. Estimates of numbers of children, cumulative and mean breastfeeding duration, and combined age at first pregnancy, and number of children were computed among parous women. ₹ Results were from the trend analysis using the categorical risk factor as a trend. ${ }^{a}$ Multivariable modeling analysis without adjusting for age at menopause. "Women who reported never pregnant, never gave birth, and had no child were grouped as "Nulliparous" in modeling analyses. ' Multivariable modeling analysis without adjusting for mean breastfeeding duration per child. BMI, body mass index; $\mathrm{Cl}$, confidence interval; HER2, human epidermal growth factor receptor-2; OR, odds ratio; $Q$, quartile

risk of ER-negative tumors in AMBER or was not associated with ER-negative $\mathrm{BC}$ in the NCI cohort consortium. Notably, the association with BMI observed in our study was mostly driven by HER2 status rather than by TNBC, which is more similar to the findings in the Malaysian study [26].

The strength of our study includes representation of BC cases from multiple hospitals in Kenya, wellannotated risk factor questionnaire and clinical data, and centralized high-quality biomarker assessment in a unique east African population.

This study was limited by the retrospective collection of risk factor data and possible reverse causation, as well as the case-only design, which prohibited us from estimating relative risks associated with each risk factor. Further, despite being the largest BC study of this type conducted in Kenya, the sample size was still relatively small to evaluate risk factors in rare tumor subtypes, especially in age-stratified analyses.

\section{Conclusion}

In summary, our findings, based on data from an indigenous African population with unique risk factor profiles, add to the growing body of knowledge regarding the etiologic heterogeneity of breast cancer molecular subtypes among geographically diverse ethnic groups. Further investigations of genetic and environmental factors that modify breast cancer risk in African populations are recommended. Inclusion of diverse regional population groups from sub-Saharan Africa in global breast cancer studies may help provide a better understanding of the subtype-specific breast cancer risk etiology, which will be critical for the development of risk prediction models in African populations.

\footnotetext{
Abbreviations

AKU: Aga Khan University; AMBER: African American Breast Cancer Epidemiology and Risk; ASR: Age-standardized rates; BC: Breast cancer; BMI: Body mass index; CA: Caucasian-American; Cl: Confidence interval; CK: Cytokeratin; EGFR: Epidermal growth factor receptor; ER: Estrogen receptor; FISH: Fluorescence in situ hybridization; HDI: Human development index; HER2: Epidermal growth factor receptor 2; HREC: Health Sciences Research Ethics Committee; IHC: Immunohistochemistry; IQR: Interquartile range; NACOSTI: National Commission for Science Technology and Innovation; PGH: Provincial General Hospital; PR: Progesterone receptor; Q1: Quartile 1; Q2: Quartile 2; Q3: Quartile 3; Q4: Quartile 4; REC: Research Ethics Committees; RS: Relative survival; SAS: Statistical software; SD: Standard deviation; SSA: Sub-Saharan Africa; TBCCC: Tianjin Cohort of Breast Cancer Cases; TDLU: Terminal duct lobular unit; TN: Triple-negative; TNBC: Triplenegative breast cancer; UCT: University of Cape Town
} 


\section{Supplementary Information}

The online version contains supplementary material available at https://doi. org/10.1186/s13058-021-01446-3.

\begin{abstract}
Supplementary Table 1.. Classifications of the five hospital groups
Supplementary Table 2. Associations between breast cancer risk factors and PR and HER2 status in Kenyan breast cancer patients $(N=838)$
\end{abstract}

Supplementary Table 3. Associations of key risk factors with ER status by hospitals ( $N=838)$

Supplementary Table 4. Associations between BMI and HER2 status stratified by hospitals $(N=821)$

Supplementary Table 5. Associations between key risk factors and ER status by age at diagnosis $(N=834)$

Supplementary Table 6. Associations between breast cancer risk factors and tumor molecular subtypes in Kenyan breast cancer patients $(N=776)$

Supplementary Table 7.. Associations of key risk factors and tumor subtypes without applying tumor grade to tumor subtype classification

Supplementary Table $\mathbf{8}$. Associations of key risk factors and tumor subtypes after excluding women missing for ki67

Supplementary Table 9.. Associations between age at first pregnancy and ER status in parous women

Supplementary Figure 1.. Associations between BMI and HER2 status stratified by hospital groups

\section{Acknowledgements}

The authors would like to acknowledge Dr. Gretchen Gierach (Division of Cancer Epidemiology \& Genetics, National Cancer Institute, USA) for her encouragement and support in the actualization of this manuscript, Angela Mutuku, Subash Govender, Johnstone Ngao, Raymond Kriel, and Veronica Ngundo for providing technical services. Permission to acknowledge all those mentioned in the Acknowledgements section was taken. JDF/SS/FM acknowledges funding from UKRI grant reference MR/S015027/1.

\section{Authors' contributions}

SS, SF, and XRY analyzed and interpreted the patient, tumor, and risk factor data as well as drafted the manuscript. RW, PB, RO, MM, FWN, RC, AJS, and SM recruited and provided patient data and clinical information. SS and ZM performed the histological and immunohistochemical examination of all BC tissues. SF performed all statistical analyses. DG, JDF, MS, PB, ZM, AS, FM, KG, $\mathrm{RN}$, and PM reviewed and provided critical input to the manuscript. All authors contributed to data collection and clinical input. All authors have read and approved the final manuscript.

\section{Funding}

The current study was funded from the Deans Fund at Aga Khan University Hospital, Nairobi. The funding sponsor did not have any role in the design of the study and collection, analysis, and interpretation of data, and in writing the manuscript. The initial study was supported through the 2011-GSK-ERI/ GlaxoSmithKline/International. The research was partially supported by the intramural research program of the Division of Cancer Epidemiology \& Genetics, National Cancer Institute, USA.

\section{Availability of data and materials}

The datasets used and/or analyzed during the current study are available from the corresponding author on reasonable request.

\section{Declarations}

\section{Ethics approval and consent to participate}

This study was nested within a previously completed breast cancer study from Kenya [13]. Ethics approval was provided by the Research Ethics Committees of the Aga Khan University Hospital Nairobi (2016/REC-32 (v3) and the Faculty of Health Sciences Research Ethics Committee of the University of Cape Town (HREC 427/2016). The study was also permitted by the National Commission for Science Technology and Innovation, Kenya (Ref No: NACOSTI/P/19/72237/28785), License number: NACOSTI/P/19/993).
Consent for publication

Not applicable

\section{Competing interests}

The authors declare that they have no competing interests.

\section{Author details}

${ }^{1}$ Department of Pathology, Aga Khan University, Nairobi, Kenya. ${ }^{2}$ University of Cape Town, Cape Town, South Africa. ${ }^{3}$ National Cancer Institute of the National Institutes of Health (NCI/NIH) Bethesda, Maryland, Rockville, USA. ${ }^{4}$ AIC Kijabe Hospital, Kijabe, Kenya. ${ }^{5}$ The University of Edinburgh, Edinburgh, UK. ${ }^{6}$ Mount Kenya University, Thika, Kenya. ${ }^{7}$ Columbia University Medical Centre, New York, USA. ${ }^{8}$ St. Mary's Mission Hospital, Nairobi, Kenya. ${ }^{9}$ Kisii University, Kisii, Kenya. ${ }^{10}$ Nyeri Provincial General Hospital, Nyeri, Kenya.

${ }^{11}$ University of the Witwatersrand, Johannesburg, South Africa. ${ }^{12}$ PathCare, Cape Town, South Africa.

Received: 25 February 2021 Accepted: 7 June 2021

Published online: 26 June 2021

\section{References}

1. Ferlay J, Soerjomataram I, Dikshit R, Eser S, Mathers C, Rebelo M, et al. Cancer incidence and mortality worldwide: Sources, methods and major patterns in GLOBOCAN 2012. Int J Cancer. 2015;136(5):E359-86.

2. Joko-Fru WY, Miranda-Filho A, Soerjomataram I, Egue M, Akele-Akpo MT, $\mathrm{N}^{\prime}$ da $\mathrm{G}$, et al. Breast cancer survival in sub-Saharan Africa by age, stage at diagnosis and human development index: A population-based registry study. Int J Cancer. 2020;146(5):1208-18.

3. McCormack V, McKenzie F, Foerster M, Zietsman A, Galukande M, Adisa C, et al. Breast cancer survival and survival gap apportionment in sub-Saharan Africa (ABC-DO): a prospective cohort study. Lancet Glob Heal [Internet]. 20201 [cited 2021 Jan 9];8(9):e1203-12. Available from: www.thelancet. com/lancetgh

4. Ferlay J, Colombet M, Soerjomataram I, Mathers C, Parkin DM, Piñeros M, et al. Estimating the global cancer incidence and mortality in 2018: GLOBOCAN sources and methods. Int J Cancer. 2019;144(8):1941-53.

5. Sayed S, Moloo Z, Ngugi A, Allidina A, Ndumia R, Mutuiri A, et al. Breast camps for awareness and early diagnosis of breast cancer in countries with limited resources: A multidisciplinary model from Kenya. Oncologist [Internet]. 2016;21(9):1138-48 Available from: https://onlinelibrary.wiley.com/ doi/abs/10.1634/theoncologist.2016-0004.

6. Knaul FM, Bhadelia A, Gralow J, Arreola-ornelas H, Langer A, Frenk J. Meeting the emerging challenge of breast and cervical cancer in low- and middle-income countries. Int J Gynecol Obstet. 2012;119:S85-8.

7. Eng A, McCormack V, dos Santos Silva I. Receptor-Defined Subtypes of Breast Cancer in Indigenous Populations in Africa: A Systematic Review and Meta-Analysis. PLoS Med [Internet]. 20141 [cited 2021 Jan 9];11(9). Available from: https://pubmed.ncbi.nlm.nih.gov/25202974/

8. Yang XR, Sherman ME, Rimm DL, Lissowska J, Brinton LA, Peplonska B, et al. Differences in risk factors for breast cancer molecular subtypes in a population-based study. Cancer Epidemiol Biomarkers Prev [Internet]. 2007 Mar [cited 2021 Jan 19];16(3):439-43. Available from: https://pubmed.ncbi. nlm.nih.gov/17372238/

9. Martínez ME, Cruz Gl, Brewster AM, Bondy ML, Thompson PA. What can we learn about disease etiology from case-case analyses? Lessons from breast cancer [Internet]. Vol. 19, Cancer Epidemiology Biomarkers and Prevention. Cancer Epidemiol Biomarkers Prev; 2010 [cited 2021 Feb 20]. p. 2710-4. Available from: https://pubmed.ncbi.nlm.nih.gov/20870734/

10. Wang S, Ogundiran T, Ademola A, Olayiwola OA, Adeoye A, Sofoluwe A, et al. Development of a breast cancer risk prediction model for women in Nigeria. Cancer Epidemiol Biomarkers Prev [Internet]. 20181 [cited 2021 Jan 9];27(6):636-43. Available from: https://pubmed.ncbi.nlm.nih.gov/29678902/

11. Tishkoff SA, Reed FA, Friedlaender FR, Ranciaro A, Froment A, Hirbo JB, et al. The Genetic Structure and History of Africans and African Americans. 2010; 324(5930):1035-44.

12. Sayed S, Moloo Z, Wasike R, Bird P, Oigara R, Govender D, et al. Is breast cancer from Sub Saharan Africa truly receptor poor? Prevalence of ER / PR / HER2 in breast cancer from Kenya. The Breast [Internet]. 2014;23(5):1-6 Available from: https://doi.org/10.1016/j.breast.2014.06.006. 
13. Sayed S, Moloo Z, Wasike R, Bird P, Oigara R, Njoroge FW, et al. Ethnicity and breast cancer characteristics in Kenya. Breast Cancer Res Treat. 2018; 167(2):425-37.

14. Wolff AC, McShane LM, Hammond MEH, Allison KH, Fitzgibbons P, Press MF, et al. Human epidermal growth factor receptor 2 testing in breast cancer: American Society of Clinical Oncology/College of American Pathologists Clinical Practice Guideline Focused Update [Internet]. Vol. 142, Archives of Pathology and Laboratory Medicine. College of American Pathologists; 2018 [cited 2021 Jan 30]. p. 1364-82. Available from: https://pubmed.ncbi.nlm.nih. gov/29846104/

15. Harbeck N, Thomssen C, Gnant M. St. Gallen 2013: Brief preliminary summary of the consensus discussion. Breast Care. 2013;8(2):102-9.

16. Bustreo S, Osella-Abate S, Cassoni P, Donadio M, Airoldi M, Pedani F, et al. Optimal Ki67 cut-off for luminal breast cancer prognostic evaluation: a large case series study with a long-term follow-up. Breast Cancer Res Treat. 2016; 157(2):363-71.

17. Goldhirsch A, Wood WC, Coates AS, Gelber RD, Thürlimann B, Senn HJ. Strategies for subtypes-dealing with the diversity of breast cancer: Highlights of the St Gallen international expert consensus on the primary therapy of early breast cancer 2011. Ann Oncol. 2011;22(8):1736-47.

18. Maisonneuve P, Disalvatore D, Rotmensz N, Curigliano G, Colleoni M, Dellapasqua $S$, et al. Proposed new clinicopathological surrogate definitions of luminal A and luminal B (HER2-negative) intrinsic breast cancer subtypes. Breast Cancer Res. 2014;16(3):R65.

19. Figueroa JD, Davis Lynn BC, Edusei L, Titiloye N, Adjei E, Clegg-Lamptey JN, et al. Reproductive factors and risk of breast cancer by tumor subtypes among Ghanaian women: A population-based case-control study. Int J Cancer. 2020;147(6):1535-47.

20. Palmer JR, Boggs DA, Wise LA, Ambrosone CB, Adams-Campbell LL, Rosenberg L. Parity and Lactation in Relation to Estrogen Receptor Negative Breast Cancer in African American Women. Cancer Epidemiol Biomarkers Prev. 2011;20(9):1883-91.

21. Palmer JR, Viscidi E, Troester MA, Hong CC, Schedin P, Bethea TN, et al. Parity, lactation, and breast cancer subtypes in African American Women: Results from the AMBER Consortium. J Natl Cancer Inst [Internet]. 2014 [cited 2020 Sep 13];106(10).

22. Bandera EV, Chandran U, Hong C, Melissa A, Traci N, Lucile L, et al. Obesity, bodyfat distribution,and riskof breast cancer subtypes in African American women participating in the AMBER consortium. Curr Drug Targets Immune Endocr Metabol Disord. 2016:150(3):655-66.

23. Ambrosone CB, Zirpoli G, Hong CC, Yao S, Troester M, Bandera E, et al Important Role of Menarche in Development of Estrogen ReceptorNegative Breast Cancer in African American Women. J Natl Cancer Inst. 2015;107(9):1-7.

24. Anderson WF, Pfeiffer RM, Wohlfahrt J, Ejlertsen B, Jensen MB, Kroman $N$. Associations of parity-related reproductive histories with $E R \pm$ and HER2 \pm receptor-specific breast cancer aetiology [Int J Epidemiol, 46, 1 (2016) (86-95)] DOl:https://doi.org/10.1093/ije/dyw286. Int J Epidemiol. 2017:46(1):373.

25. Fortner RT, Sisti J, Chai B, Collins LC, Rosner B, Hankinson SE, et al. Parity, breastfeeding, and breast cancer risk by hormone receptor status and molecular phenotype: results from the Nurses' Health Studies. Breast Cancer Res. 2019;21(1):40.

26. Abubakar M, Sung H, Devi B, Guida J, Tang T, Pfeiffer R, et al. Breast cancer risk factors, survival and recurrence, and tumor molecular subtype: Analysis of 3012 women from an indigenous Asian population. Breast Cancer Res. 2018;20(1):1-14

27. Chatterjee, Nimrat Walker G. Pooled analysis of nine cohorts reveals breast cancer risk factors by tumor molecular subtype. Physiol Behav [Internet]. 2017;176(10):139-48. Available from: file:///C:/Users/Carla Carolina/Desktop/ Artigos para acrescentar na qualificação/The impact of birth weight on cardiovascular disease risk in the.pdf

28. Chen L, Li Cl, Tang M-TC, Porter P, Hill DA, Wiggins CL, et al. Reproductive factors and risk of luminal, HER2-overexpressing, and triple-negative breast cancer among multiethnic women. Cancer Epidemiol Biomarkers Prev. 2016; 25(9):1297-304.

29. Ma H, Bernstein L, Pike MC, Ursin G. Reproductive factors and breast cancer risk according to joint estrogen and progesterone receptor status: A meta-analysis of epidemiological studies. Breast Cancer Res [Internet]. 2006 [cited 2020 Dec 17];8(4). Available from: https://pubmed. ncbi.nlm.nih.gov/16859501/
30. Islami F, Liu Y, Jemal A, Zhou J, Weiderpass E, Colditz G, et al. Breastfeeding and breast cancer risk by receptor status - a systematic review and metaanalysis. Ann Oncol. 2015;26(12):2398-407.

31. Tan MM, Ho WK, Yoon SY, Mariapun S, Hasan SN, Shin-Chi Lee D, et al. A case-control study of breast cancer risk factors in 7,663 women in Malaysia. PLoS One. 2018;13(9):e0203469.

32. Nindrea RD, Aryandono T, Lazuardi L. Breast cancer risk from modifiable and non-modifiable risk factors among women in Southeast Asia: A metaanalysis. Asian Pacific J Cancer Prev. 2017;18(12):3201-6.

33. Ghoncheh M, Pournamdar Z, Salehiniya H. Incidence and mortality and epidemiology of breast cancer in the world. Asian Pacific J Cancer Prev. 2016;17(sup3):43-6.

34. Sherman ME, Rimm DL, Yang XR, Chatterjee N, Brinton LA, Lissowska J, et al. Variation in breast cancer hormone receptor and HER2 levels by etiologic factors: A population-based analysis. Int J Cancer [Internet]. 20071 [cited 2021 Jan 9];121(5):1079-85. Available from: http://doi.wiley.com/10.1002/ ijc.22812

\section{Publisher's Note}

Springer Nature remains neutral with regard to jurisdictional claims in published maps and institutional affiliations.
Ready to submit your research? Choose BMC and benefit from:

- fast, convenient online submission

- thorough peer review by experienced researchers in your field

- rapid publication on acceptance

- support for research data, including large and complex data types

- gold Open Access which fosters wider collaboration and increased citations

- maximum visibility for your research: over $100 \mathrm{M}$ website views per year

At BMC, research is always in progress.

Learn more biomedcentral.com/submissions 